\title{
Classification of Puck Possession Events in Ice Hockey
}

\author{
by \\ Moumita Roy Tora \\ B.Sc, Brac University, 2014 \\ A THESIS SUBMITTED IN PARTIAL FULFILLMENT \\ OF THE REQUIREMENTS FOR THE DEGREE OF \\ Master of Science \\ in \\ THE FACULTY OF GRADUATE AND POSTDOCTORAL \\ STUDIES \\ (Computer Science)
}

The University of British Columbia

(Vancouver)

September 2017

(C) Moumita Roy Tora, 2017 


\section{Abstract}

Group activity recognition in sports is often challenging due to the complex dynamics and interaction among the players. In this thesis, we propose a deep architecture to classify puck possession events in ice hockey. Our model consists of three distinct phases: feature extraction, feature aggregation and, learning and inference. For the feature extraction and aggregation, we use a Convolutional Neural Network $(\mathrm{CNN})$ followed by a late fusion model on top to extract and aggregate different types of features that includes handcrafted homography features for encoding the camera information. The output from the CNN is then passed into a Recurrent Neural Network (RNN) for the temporal extension and classification of the events. The proposed model captures the context information from the frame features as well as the homography features. The individual attributes of the players and the interaction among them is also incorporated using a pre-trained model and team pooling. Our model requires only the player positions on the image and the homography matrix and does not need any explicit annotations for the individual actions or player trajectories, greatly simplifying the input of the system. We evaluate our model on a new Ice Hockey Dataset and a Volleyball Dataset. Experimental results show that our model produces promising results on both these challenging datasets with much simpler inputs compared with the previous work. 


\section{Lay Summary}

Group activity recognition is the task of determining what a group of people are doing given a single image or a short clip of video. We have looked at group activity recognition in sports videos, particularly ice hockey. Thus given a sequence of images, we aim to classify the sequence into a group activity or event. There are many possible events that can happen in ice hockey but we have looked at a subset of only those events which involve the possession of the puck by the players. Some examples include pass and shot. We have solved this problem by proposing a deep network architecture which takes into account player appearance and contextual information. These features from different sources are fused together and passed into a temporal model to learn the dependencies across the images in the given sequence. 


\section{Preface}

This thesis is submitted in partial fulfillment of the requirements for a Master of Science Degree in Computer Science. The entire work presented here is original work done by the author, Moumita Roy Tora, performed under the supervision of Professor James J. Little. A version of this work has appeared in the following publication:

- "Classification of Puck Possession Events in Ice Hockey", The IEEE Conference on Computer Vision and Pattern Recognition (CVPR) Workshops, Jul 2017, pp. 91-98. 


\section{Table of Contents}

Abstract .......................... ii

Lay Summary $\ldots \ldots \ldots \ldots \ldots$ iii

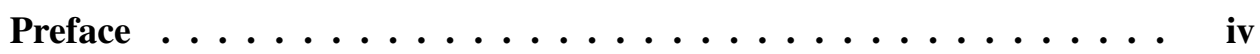

Table of Contents $\ldots \ldots \ldots \ldots \ldots \ldots \ldots$

List of Tables $\ldots \ldots \ldots \ldots \ldots \ldots \ldots \ldots \ldots$ vii

List of Figures $\ldots \ldots \ldots \ldots \ldots$ viii

Acknowledgments $\ldots \ldots \ldots \ldots \ldots$ xiii

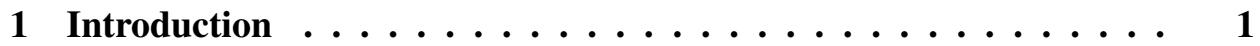

2 Related Work . . . . . . . . . . . . . . . 8

2.1 Group Activity Recognition . . . . . . . . . . . . . . 8

2.2 Group Activity/ Event Classification in Sports . . . . . . . . . . 9

2.2.1 Formulating Ice Hockey Events . . . . . . . . . . . 9

2.2.2 Analogy of Our Problem with Other Team Sports . . . . . 10

2.3 Deep Learning . . . . . . . . . . . . . . . . . . . . . 12

3 Overview of the Methodologies . . . . . . . . . . . . . 13

3.1 Convolutional Neural Network $(\mathrm{CNN}) \ldots \ldots \ldots$

3.1 .1 Evolution of CNNs . . . . . . . . . . . . 15 
3.2 Recurrent Neural Network $(\mathrm{RNN}) \ldots \ldots \ldots$

4 Our Method $\ldots \ldots \ldots \ldots \ldots$

4.1 Individual Attributes . . . . . . . . . . . . . . 18

4.2 Contextual Information from the Image . . . . . . . . . . . . 19

4.3 Homography for the Spatial Feature Representation . . . . . . . . 20

4.3.1 Computing Features from the Homography Matrix . . . . 20

4.4 Fusion Model for Combining Homography Features and Frame Features . . . . . . . . . . . . . . . 22 2

4.5 Temporal Model . . . . . . . . . . . . . . . 22

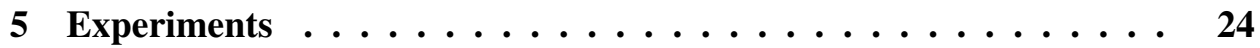

5.1 Evaluation Metric . . . . . . . . . . . . . . . 24

5.2 Baselines ...................... 25

5.3 Implementation Details . . . . . . . . . . . . . . . 27

5.4 Ice Hockey Dataset . . . . . . . . . . . . . . . . . . . . . . . . . . . . . . . .

$5.4 .1 \quad$ Event Annotation . . . . . . . . . . . . . . 28

5.4 .2 Tracking Data . . . . . . . . . . . . . 28

5.4.3 Homography Transformation . . . . . . . . . . . . 29

5.4.4 Quantitative Results . . . . . . . . . . . . . . . . . 29

5.4.5 Qualitative Results .............. 36

5.5 Volleyball Dataset . . . . . . . . . . . . . . . . . 37

5.5.1 Quantitative Results . . . . . . . . . . . 37

5.5.2 Qualitative Results ............. 38

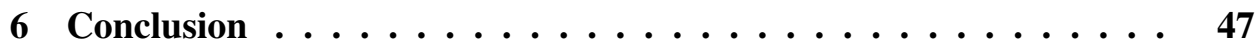

Bibliography ............................ 49 


\section{List of Tables}

Table 5.1 Event descriptions and corresponding number of training examples in the Ice Hockey Dataset. . . . . . . . . . . . . . . 30

Table 5.2 The descriptions of the baselines. . . . . . . . . . . . 30

Table 5.3 Performance of our model on Ice Hockey Dataset compared to the baselines. In the fine-tuning column, w/o and $w$ represent without and with fine-tuning, respectively.

Table 5.4 Per class recall and precision of our model on the Ice Hockey Dataset. . . . . . . . . . . . . . . . .

Table 5.5 Event descriptions in the Volleyball Dataset. In the original dataset and all our experiments, these events are further classified into right and left where right always refers to the team in the right side of the net and left refers to the team in the left side. For example, r-pass refers to the pass event occurring in the right side.

Table 5.6 The Descriptions of the baselines. . . . . . . . . . . . . .

Table 5.7 Performance of our model on Volleyball Dataset compared to the baselines and previous work. $w$ and w/o refers to with and without fine-tuning respectively. . . . . . . . . . . . . .

Table 5.8 Per class recall and precision of our model on the Volleyball Dataset. . . . . . . . . . . . . . . . . 


\section{List of Figures}

Figure 1.1 Example images of group activity. . . . . . . . . . . .

Figure 1.2 Example images of puck possession events. In each image, the dashed red line is the potential trajectory of the puck except for LPR in which the red lines are the trajectories of potential player movements. . . . . . . . . . . . .

Figure 1.3 Schematics of puck possession events. This figure shows schematics of five puck possession events that our system aims to classify. In some events, individual players appearances/motions could be very similar such as dump in and dump out. . . . . .

Figure 1.4 CNN model with a late fusion on top. $f c$ corresponds to fully connected convolutional layer. $f c 7$ is the second last fully connected layer before the classifier in AlexNet. The numbers by the arrows correspond to the input/output feature vector dimensions. We have fine-tuned last two layers of AlexNet on our datasets. . . . . . . . . . . . . . . . . . .

Figure 1.5 Overview of our model. Our method can classify puck possession events for ice hockey games from a sequence of images. We first extract scene features from a whole frame, individual features from the players and homography features using the homography matrix. Team pooling is performed for the player features and all the features are then fused. Finally we train a BLSTM model using a sequence of temporal features. . . . . 
Figure 3.1 AlexNet illustration. The input is a 224 by 224 image that goes through several hidden layers before being classified by softmax in the final layer. The output from the last fully connected layer is a 1000 dimensional feature vector that goes to the classifier and the output of the classifier is a score vector for each possible classes. Source: [2] . . . . . . . . . . . .

Figure 3.2 Block diagram of a simple RNN that unfolds with time forming a chain structure. $A$ refers to one unit of the RNN, $x$ and $h$ refer to the input and the output at a given time step. The hidden state values of one unit are passed to the next one sequentially and are represented by the arrow. Source: [5] . . .

Figure 3.3 Building Block of an LSTM unit. Each LSTM unit has 3 gatesinput, output and forget, the input block and the output block. There are different types of weights associated with each component of the unit which are learned during training the network. The gates are usually controlled by sigmoid functions. Source: $[4] \ldots \ldots \ldots \ldots \ldots \ldots$

Figure 4.1 Weight computation method for a player and his associated centers. . . . . . . . . . . . . . . .

Figure 5.1 The transformation from the image to the template coordinate system by the homography matrix. . . . . . . . . . . 29

Figure 5.2 Training loss over time for the Ice Hockey Dataset. . . . . . . 31

Figure 5.3 Training accuracy over time for the Ice Hockey Dataset. . . . 
Figure 5.4 Confusion matrix for top-1 event prediction with a tolerance of 0.0 on the Ice Hockey Dataset. The rightmost column shows the precision and the bottom row shows the recall per class in percentages. The green values represent the percentages of the correct predictions by the model whereas the red values show the percentages of the incorrect predictions. For example, recall for $l p r$ is $39 \%$ and the its precision is $46.6 \%$ which means out of all the $l p r$ events, $39 \%$ were correctly classified and out of all the $l p r$ predictions made by the model, $46.6 \%$ were correct. The blue rectangle in the bottom right corresponds to the overall accuracy. . . . . . . . . . . . .

Figure 5.5 Confusion matrix for top-2 event prediction with a tolerance of 0.15 on the Ice Hockey Dataset. The rightmost column shows the precision and the bottom row shows the recall per class in percentages. The green values represent the percentages of the correct predictions by the model whereas the red values show the percentages of the incorrect predictions. For example, recall for $l p r$ is $74.3 \%$ and the its precision is $78 \%$ which means out of all the $l p r$ events, $74.3 \%$ were correctly classified and out of all the $l p r$ predictions made by the model, $78 \%$ were correct. The blue rectangle in the bottom right corresponds to the overall accuracy. . . . . . . . . . . . . . . 33

Figure 5.6 Training loss over time for the Volleyball Dataset. . . . . . . . 40

Figure 5.7 Training accuracy over time for the Volleyball Dataset. . . . . 40 
Figure 5.8 Confusion matrix for top-1 event prediction with a tolerance of 0.0 on the Volleyball Dataset. The rightmost column shows the precision and the bottom row shows the recall per class in percentages. The green values represent the percentages of the correct predictions by the model whereas the red values show the percentages of the incorrect predictions. For example, recall for $r$-set is $41.7 \%$ and the its precision is $51.3 \%$ which means out of all the $r$-set events, $41.7 \%$ were correctly classified and out of all the $r$-set predictions made by the model, $51.3 \%$ were correct. The blue rectangle in the bottom right corresponds to the overall accuracy. . . . . . . . . . .

Figure 5.9 Confusion matrix for top-2 event prediction with a tolerance of 0.15 on the Volleyball Dataset. The rightmost column shows the precision and the bottom row shows the recall per class in percentages. The green values represent the percentages of the correct predictions by the model whereas the red values show the percentages of the incorrect predictions. For example, recall for $r$-set is $59.9 \%$ and the its precision is $70.9 \%$ which means out of all the $r$-set events, 59.9\% were correctly classified and out of all the $r$-set predictions made by the model, $70.9 \%$ were correct. The blue rectangle in the bottom right corresponds to the overall accuracy. . . . . . . . . . . .

Figure 5.10 Qualitative results. The correct predictions made by our model with a tolerance of 0.0 on the Ice Hockey Dataset. . . . . . . .

Figure 5.11 Qualitative results. The correct predictions made by our model with a tolerance of 0.15 on the Ice Hockey Dataset. True refers to the actual class, Pred 1 and Pred 2 refer to the prediction with a tolerance of 0.0 and 0.15 respectively and $\delta$ refers to the difference in probabilities between the two predictions.

Figure 5.12 Qualitative results. The images show the cases where our model failed on the Ice Hockey Dataset. . . . . . . . . . . . . .

Figure 5.13 Qualitative results. The correct predictions made by our model with a tolerance of 0.0 on the Volleyball Dataset. . . . . . . . 
Figure 5.14 Qualitative results. The correct predictions made by our model with a tolerance of 0.15 on the Volleyball Dataset. True refers to the actual class, Pred 1 and Pred 2 refer to the prediction with a tolerance of 0.0 and 0.15 respectively and $\delta$ refers to the difference in probabilities between the two predictions.

Figure 5.15 Qualitative results. The figures show the cases where our model failed on the Volleyball Dataset. . . . . . . . . . . . . . . 


\section{Acknowledgments}

I would like to express my greatest gratitudes to a number of people who have been a continuous source of support during my Masters.

First of all I would like to thank my supervisor, Professor James J. Little, who is not only a great mentor but also one of the kindest human beings I have come across. From the very beginning of my research, he has been very supportive about my work and interests. He appreciated and acknowledged every little idea that I came up with and he helped me to gain new insights of any problem. I would also like to thank Professor Leonid Sigal for agreeing to read my thesis as a second reader.

Next, I would like to thank University of British Columbia (UBC) for giving me a lifetime opportunity to enhance my knowledge. I have enjoyed every little piece of my 2 years here. There were times when I was too exhausted with all the courses I had to take but at the end of the day, I gained a new perspective of my life. I would like to thank all the great instructors I have come across, my peers, friends, family and all my lab-mates who always helped me whenever I needed.

This work was supported in part by the Natural Sciences and Engineering Research Council of Canada (NSERC) and the Institute for Computing, Information and Cognitive Systems (ICICS) at UBC, and enabled in part by WestGrid and Compute Canada. I would like to thank them for funding me and providing me the opportunity to travel to a number of conferences and meetings.

Lastly, my heartiest gratefulness is for my parents, brother and husband who believed in me more than myself. My parents taught me to dream bigger from my very childhood and whatever little I have learned in my life is only because of their inspiration and blessings. 


\section{Chapter 1}

\section{Introduction}

Computer vision has been widely used in many sports applications [43]. The number of applications has expanded from information extraction such as player detection and tracking [38] to new visual information generation such as free-viewpoint video generation [25], and further to prediction of shot location [63] and broadcast camera angle planning [12].

Among various applications, group activity recognition is an active research area. Group activity recognition refers to determining what a group of people are doing, providing semantic and abstract descriptions for a sequence of images. Figure 1.1 shows some examples of group activities collected from Collective Activity Dataset [15]. Group activity recognition is similar to activity/action recognition but additional dynamics of the group interaction needs to be considered in determining group activities. In most group activities, the human-human or the human-object interaction need to be modeled. In order to do that, we need to detect/segment the humans and the objects first which is not trivial. For video classification, additional cues such as poses, tracking, motion and camera movements play important roles.

However, sports activity recognition can be very complex due to the rapid transition between the events, occlusions and fast movements of the players, varied camera viewpoints, and camera motions. Moreover, spatiotemporal structures of events vary greatly in different sports. For example, the locations of the players in volleyball is relatively static compared to players in ice hockey. Another key challenge is to distinguish between motion, action, activity and event in sports because 

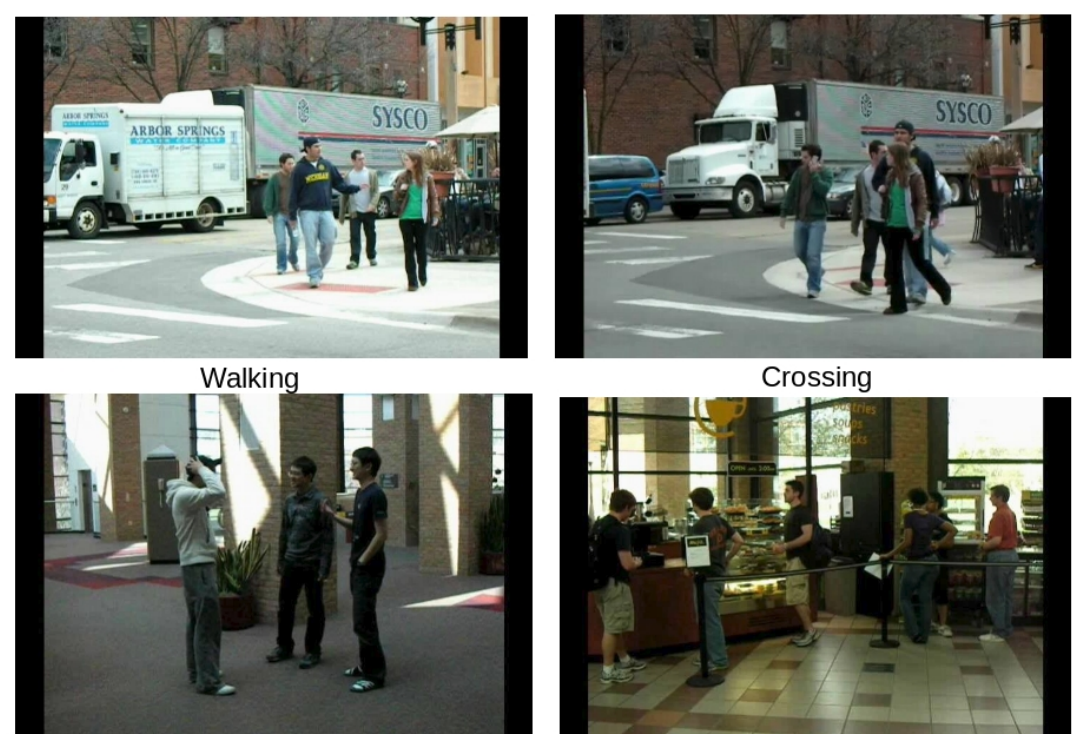

Talking

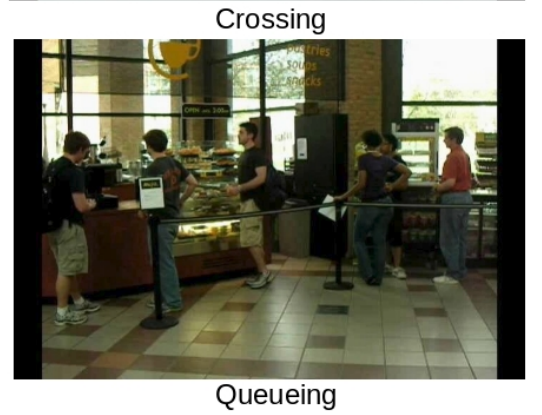

Figure 1.1: Example images of group activity.

the transition between these four states is very rapid and complex. Our focus is on events classification. In ice hockey, sometimes the events are instants of just 1 frame whereas other times they can be much longer. Sometimes there is a long interval between two events and there is no clear boundary between them. There are also cases where two events can occur simultaneously on the same frame. All these factors make the recognition problem complex and hard to generalize across different sports. Most previous work has tried to address group activity classification in non-sports applications [7, 13, 14, 36]. Consequently, studying a particular sport with domain knowledge is valuable and complementary to general activity recognition.

We are interested in classification of puck possession events in ice hockey games. Puck possession events are those events that involve players taking over or losing the control of the puck. For example, in the pass event, the control of the puck shifts from one player to another in the same team. Unlike other ball sports 


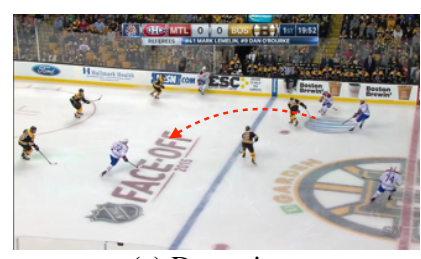

(a) Dump in

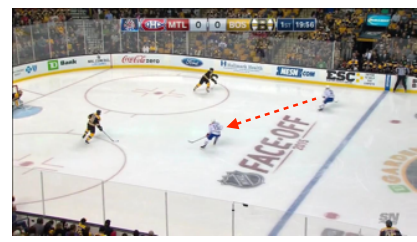

(d) Pass

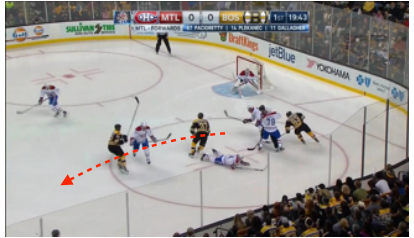

(b) Dump out

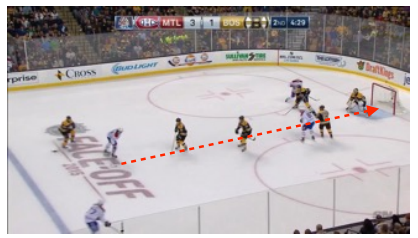

(e) Shot

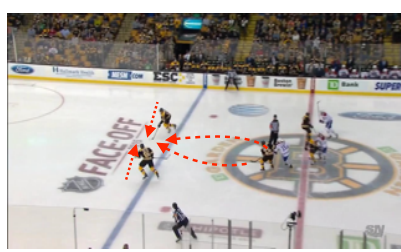

(c) LPR

Figure 1.2: Example images of puck possession events. In each image, the dashed red line is the potential trajectory of the puck except for LPR in which the red lines are the trajectories of potential player movements.

such as basketball and soccer, the puck in hockey can be in the possession of neither team for extended time periods, for example, when the puck moves out from the defensive zone or into the offensive zone. All the events we have considered involve interaction between the puck and the player. This is different from sports such as running or swimming, where there is no need to model the object-human or humanhuman interaction. However modeling this interaction in ice hockey is difficult as the puck is not very clear in most of the frames because of its size, color and rapid movement unlike sports such as volleyball or soccer where the ball is much bigger and visible. The dataset, provided by SPORTLOGiQ (http://sportlogiq.com/), contains the annotations for puck possession events in National Hockey League (NHL) games. The playing surface (rink) is large and enclosed by the boards. Play-byplay commentary, provided in real-time during a broadcast, annotates shooting, scoring and hit events at time intervals of 3 to 15 seconds, typically, while the intervals between our annotated events range from 3 to 200 frames (30 FPS). The output of our system can greatly benefit the manual events annotation with some tolerance of error. The classification results may enable coaches and analysts to determine both strategic concepts and support evaluation of individual players.

Our proposed model aims to classify five puck possession events which are dump in, dump out, pass, shot and loose puck recovery (LPR). The descriptions of 


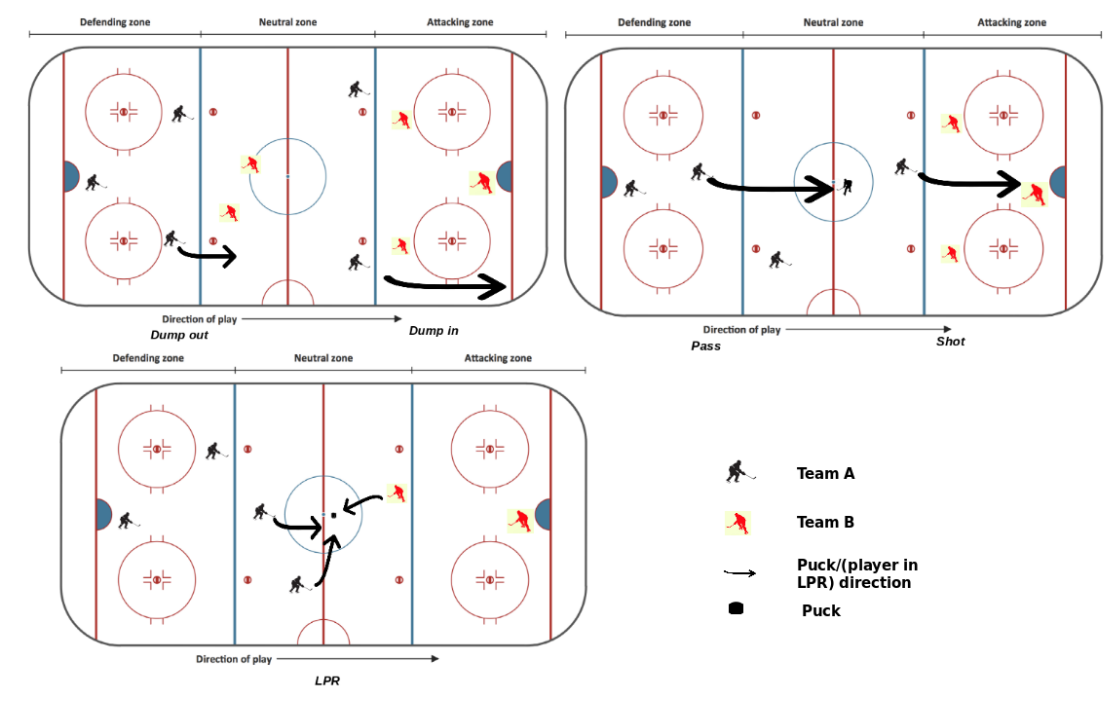

Figure 1.3: Schematics of puck possession events. This figure shows schematics of five puck possession events that our system aims to classify. In some events, individual players appearances/motions could be very similar such as dump in and dump out.

these events are in Table 5.1. In all the events but the LPR, the puck-control goes from possession to no possession of the players. In case of LPR, the puck is not in possession of any player and it goes from no possession to possession. Figure 1.2 shows example images of these events. In the images, the dashed red lines show the movements of the pucks or the players. Figure 1.3 shows the schematics of these events on the ice hockey strategy board.

An important descriptive cue for the classification of possession events is the location of the puck and the players in coordinates of playing field. However, it is extremely hard to track the puck in images as the puck is very small and moves very fast. Due to motion blur, the puck's color and texture can be merged into the backgrounds. Without any kind of puck information, the event classification problem is even more difficult.

Alternative cues for puck possession events classification are player locations in the image and their appearance, their spatiotemporal information and estimating player locations in the playing ground coordinates. Players are coached to keep the 
team shape and move to offense/defense together. But looking only at individual players can be ambiguous in some events. For example, the player appearance and action might be very similar in the two events - pass and shot. In this case, additional cues such as context information are necessary to distinguish these two events.

Our model uses a deep architecture which requires the homography matrix, detected bounding boxes of the players and the corresponding frames. Figure 1.5 shows the pipeline of our method and Figure 1.4 shows the CNN model with a late fusion on top that we have trained. The input of our method is a sequence of images with player detection results (bounding boxes in the image) and the homography features. The detailed computation of these features is described in Section 3.3. Our method first extracts context features from the whole image, individual features from the player image patches and homography features using the homography matrices. The homography features represent the spatial distribution in rink coordinate of the players. Because the number of detected players changes over frames, we use a max pooling layer to aggregate the individual features. The max pooling is done in a team-wise fashion. Then, we use a bidirectional LSTM to train an event classification model using features from the sequence of given images.

Building upon the existing work, our model takes advantage of the discriminative power of deep learning and captures the structural and spatiotemporal information in group activities. Moreover, it shows how combining contextual information and person level features can improve accuracy for events that are very similar to each other. The main contribution of our work is four-fold. First, we propose a benchmark for event classification on a new challenging Ice Hockey Dataset. Second, we extensively study the features from whole frames and individual players. We provide solid evidence that our model works best when the individual's information is combined with the context of the events in ice hockey games. Third, we propose a new fusion approach to combine features from different sources. Lastly, we also test our method on a Volleyball Dataset and obtain reasonable results that show the generalization property of our approach across quite different sports.

The rest of the paper is organized as follows: Chapter 2 surveys previous work in sports, group activities and deep learning, Chapter 3 gives an overview of the methodologies we have used in our experiments, Chapter 4 describes our archi- 


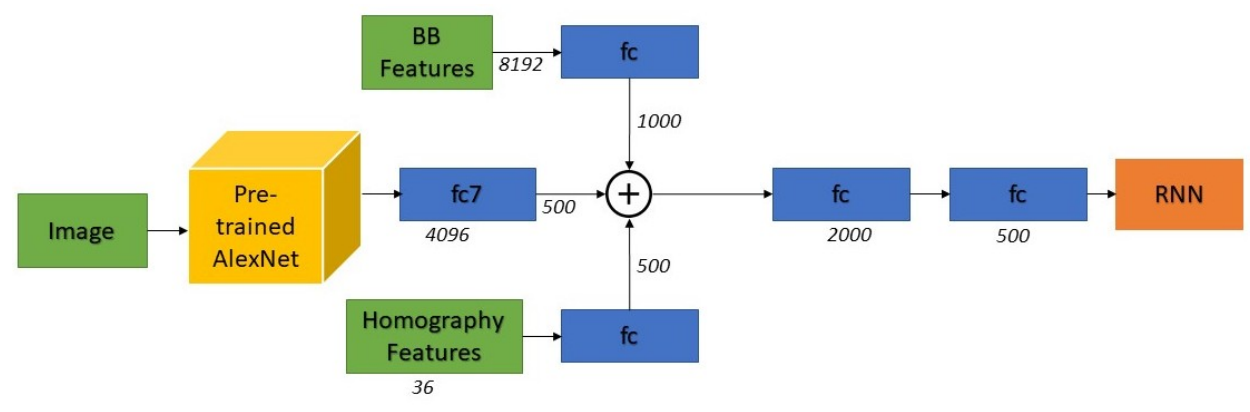

Figure 1.4: CNN model with a late fusion on top. $f c$ corresponds to fully connected convolutional layer. $f c 7$ is the second last fully connected layer before the classifier in AlexNet. The numbers by the arrows correspond to the input/output feature vector dimensions. We have fine-tuned last two layers of AlexNet on our datasets.

tecture in detail, Chapter 5 illustrates the experiments and results we have found followed by Chapter 6 that summarizes our work with suggestion of some possible extensions in the future. 


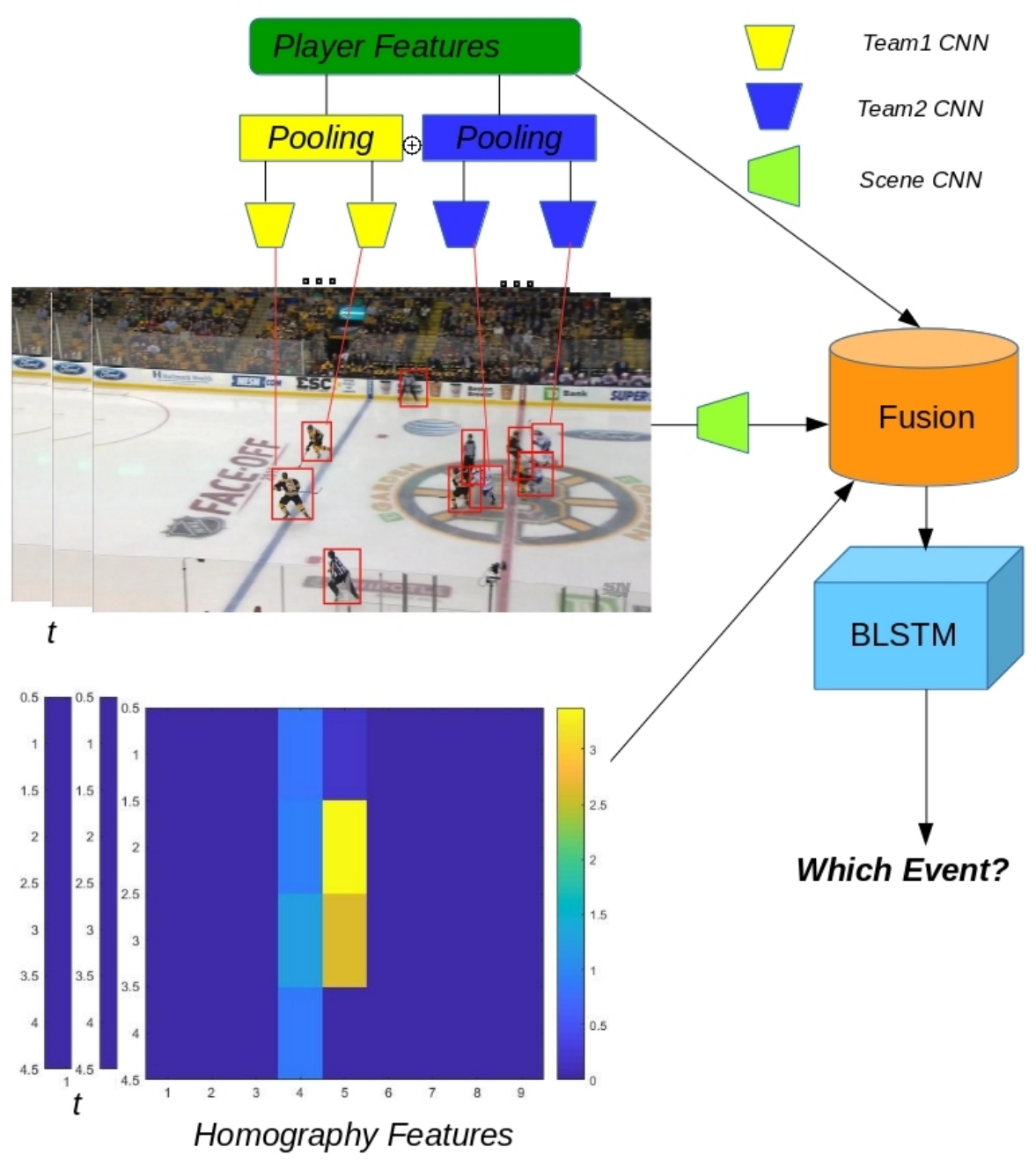

Figure 1.5: Overview of our model. Our method can classify puck possession events for ice hockey games from a sequence of images. We first extract scene features from a whole frame, individual features from the players and homography features using the homography matrix. Team pooling is performed for the player features and all the features are then fused. Finally we train a BLSTM model using a sequence of temporal features. 


\section{Chapter 2}

\section{Related Work}

\subsection{Group Activity Recognition}

Group activity recognition has been an active area of research over the years. Before the dramatic success of deep learning on a variety of tasks, researchers mostly relied on hand-crafted features [47] [15] [62]. When deep learning started gaining attention, similar to most other applications, group activities also started to take advantage of these neural networks [28] [45] [61] [8] [52]. In parallel, there was other interesting work which continued to explore handcrafted features with some notable amount of success. In most work, researchers break down the problem of group activity recognition into subproblems of tracking and identifying individual actions, key persons or interaction among the individuals. The knowledge from these smaller problems is then combined to learn the group activity [51] [14] [36] Graphical models seemed to gain particular attention in the hierarchical approach [44] [7] [35].

Besides, there are other models where researchers started to combine deep models with other machine learning approaches that can boost the performance further, because the deep models are sometimes hard to interpret and require lots of fine-tuning. Most recent work tries to explore advanced deep models such as deep reinforcement learning or deep graphical models [16] [45] [61] [8] [52] [64]. But these concepts are still very new to the domain of activity recognition. 


\subsection{Group Activity/ Event Classification in Sports}

The domain of sports analysis is vast because each sport has its unique characteristics. As a result, a model from a particular sport is unable to achieve reasonable performance on other sports datasets. However, researchers have narrowed down the domain into specific sports particularly the most challenging ones and have tried to solve different aspects over the years. For example, Yue-Hei $\mathrm{Ng}$ et al. [65] proposed and evaluated different deep neural network architectures to classify longer sequence of sports videos. Meanwhile, various approaches have been proposed for different types of sports analysis [10, 42, 49]. Although, there is some previous work on player tracking and action recognition in ice hockey [39-41], to the best of our knowledge, no previous work has been done for event classification in ice hockey.

\subsubsection{Formulating Ice Hockey Events}

Sports events can be broadly classified based on several characteristics: object vs. non-object sports such as swimming and golf, instant events vs. a sequence of actions, single person action/activities vs. team sports, game policies and so on. In our problem, all the events are centered around the puck and what the players are doing with it. So we need to consider the human-object and human-human interaction. The object-human interaction in sports can be analogous to non-sports activities such as cutting fruits with a knife. This activity can be broken down into a sequence of shorter actions such as holding the knife, holding the fruit, touching the knife with the fruit and cutting it. Modelling this less complex activity still requires attention to delicate details such as how the hand is holding the objects and what are the shapes and textures of these objects. Events in sports are ususally much more complicated sequence of actions and the transition between these actions are very fast. The actions might also look much more similar to each other and there could be multiple actions occurring on the same frame.

We can further narrow down our problem into multi-person activities. Although some of the events such as goal or shot could be considered as sole actions, determining these events often needs to look at the surrounding players and their individual actions. For example, during the shot event, the opposing team defend- 
ers will most likely try to dump the puck out. However, the duration of each event and the possession of the puck varies widely. The puck may be out of possession for a long period of time unlike other sports and there is no annotation for the frames where the puck is not in possession of any team and when an event ends.

\subsubsection{Analogy of Our Problem with Other Team Sports}

\section{Volleyball}

Ibrahim et al. [28] built a hierarchical deep network to learn the individual actions using one LSTM which is then combined with features extracted from CNNs to pass into another LSTM to predict the group actions. However, their method has difficulties on events which are very similar to each other. Very recently, Bagautdinov et al. [8] proposed a deep network that jointly detects the players, infers their social actions and estimates the collective actions. Their method outperforms [28] on the Volleyball Dataset. However, both these models need explicit labels for individual actions which are expensive and hard to label for ice hockey. In our scenario, the events are similar to each other and there is no person-level annotations. Both these factors make these models less suitable for our problem. On the other side, volleyball is very different from ice hockey games. Although both these games involve possessing the puck/ball and modeling the object-human or human-human interaction, the puck is much smaller, faster and harder to track than the ball. Moreover, the ball is mostly in the air in volleyball whereas the puck can be either in the air or on the ground. The players from the two teams in volleyball never mingle with each other and are clearly separated by a line/net but in ice hockey, they are almost never separated. The relative motions of the volleyball players are static compared to ice hockey where the players skate at a fast speed all the time and hit and bounce from the surrounding boards. If the ball goes out of the boundary in volleyball, it is usually a miss whereas in ice hockey the puck can go in and out of the boundary line anytime while the game continues. 


\section{Basketball}

Although there has been some work on event detection in basketball, much focus has not been given on the event classification problem. Ramanathan et al. [45] proposed a deep temporal model with an attention mechanism to detect and classify events on a new basketball dataset which they have made available to the public. Their model attends to a subset of players in a frame rather than all the players because usually a few players are participating in the event. Their unified approach can detect the key players and classify the activities simultaneously. However, the authors have mentioned that the performance is usually poor for classes with fewer examples. Although the overall mean average precision for their approach is slightly better than other baselines, per class accuracy is still not satisfactory for many events. They also have not experimented on any other dataset that makes it difficult to compare the generalization property of their approach. There are many fundamental differences between basketball and ice hockey. The ball is always in possession of some player during an event and it is always in the air whereas the puck may or may not be in possession of any team and can be either in the air or on the ground. The player can either hold, bounce or throw the ball with the hands whereas the puck is always handled with the sticks without any human touch. Moreover, similar to volleyball or soccer, the ball in basketball is much bigger than the puck and is generally easier to distinguish from the background. Nevertheless, each sport has its own challenges which might require the need of sport-specific precise models.

\section{Soccer}

Compared to many other sports, research using the soccer videos dates to a long time back. Kong et al. [33] proposed a local motion-based approach to classify three different actions. Their model first computes SIFT features and then relative motion descriptors from the background and the foreground key-point sets using the bag-of-words strategy. Recently, Komori et al. [59] have proposed a hierarchical deep architecture for recognizing three different activities in soccer. Other work in soccer includes field localization [27], player tracking [37], automatic camera planning [11] etc. Soccer fields are usually open and much bigger compared 
to the ice hockey rinks which are enclosed by boundaries. The movement of the players are much faster in ice hockey than soccer. The soccer ball is bigger and easier to track than the puck. But in both the sports, the ball and the puck may or may not be in possession of any of the teams and can be in the air or on the ground. The group events are also very similar in both these sports.

\subsection{Deep Learning}

As more data became available, the success of Convolutional Neural Networks (CNN) has been proved in numerous applications over the last decade on computer vision tasks such as image recognition [34] and video analysis [31, 53]. Recurrent Neural Networks (RNN) particularly Long Short Term Memory (LSTM) [26] are widely popular models that are well suited for variable length sequence inputs. LSTM has been successfully applied to speech and handwriting recognition [22], human action recognition [9, 17] and image caption generation [30, 60].

Researchers have shown different ways to combine LSTMs with CNNs or graphical models for group activity recognition. For example, Deng et al. integrated a graphical model with a deep neural network [16]. The network learns structural relations by representing individuals and the scene as nodes passing messages among them and imposing a gating mechanism to determine the meaningful edges. But the method is not designed for sports activities where interactions between individuals are generally more complicated. In [45], Ramanathan et al. argued that in many group activities redundant information can be ignored by concentrating on a subset of people who contribute to the group activity. Thus, they first extracted features from individuals who are attending to the event as well as global context features representing the entire scene and then solved the problem of event classification using a deep network. 


\section{Chapter 3}

\section{Overview of the Methodologies}

This chapter provides a high level description of the algorithms we have used. Our core architecture is built on two widely popular supervised models - a Convolutional Neural Network (CNN) and a Recurrent Neural Network (RNN). In general, each of these networks takes some feature vectors and the desired output values as inputs, and the networks are trained using a loss function. During training, the network learns the weights and the hyper-parameters from the given inputs and the trained network is then applied to an unseen dataset to map the inputs based on the learned function. However, there are some fundamental differences between the two networks and they are usually used for different purposes. For example, a CNN is used mainly for visual understanding whereas an RNN is known for its temporal structure that can learn sequential problems.

\subsection{Convolutional Neural Network (CNN)}

A convolutional neural network is a feed forward neural network which has an input, an output and multiple hidden layers of specific types. The input is a 3dimensional image, the hidden layers can be pooling, convolutional, fully connected, normalization or ReLU (rectified linear unit) and the output is usually a score for each target class (for the classification problems), all of which are stacked together to form a convolutional neural network [1]. The layers in CNN are made up of neurons that have associated weights and biases, certain hidden layers have 


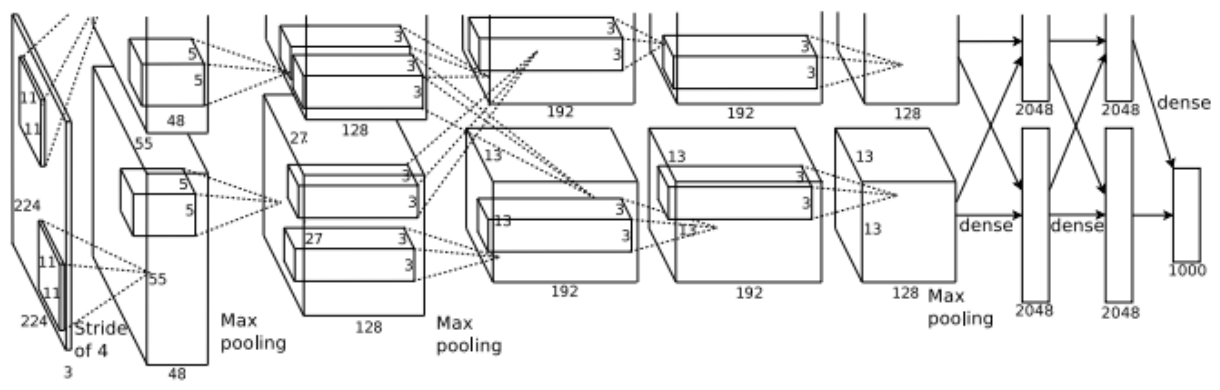

Figure 3.1: AlexNet illustration. The input is a 224 by 224 image that goes through several hidden layers before being classified by softmax in the final layer. The output from the last fully connected layer is a 1000 dimensional feature vector that goes to the classifier and the output of the classifier is a score vector for each possible classes. Source: [2]

activation functions and parameters, the last fully connected layer has a loss function and the network is trained to learn visual features from the input image by optimizing the objective function and updating the weights. During the forward pass of the training, the network predicts the output based on the current weights and during backpropagation, it computes the error and the gradient of the loss function to minimize the error which is propagated from the output back to the network updating the network weights. Figure 3.1 shows an example image of AlexNet [34] which is a widely used CNN for classification problems. The input to the network is an image sample of fixed dimension and the output is a probability score for each target class. The image goes through a series of hidden layers and passes through the classification layer, softmax after the last fully connected layer. In general, all the layers take 3D activation maps as inputs and transform them to some other activation maps by means of a differentiable function to represent higher level features. In the first layer, the input is the raw pixels of an RGB image and the output is a number of activation maps obtained by convolving the image with the filter. The number and output dimension of the activation maps depend on the number and the dimension of the filters applied to the input during convolution and the feature maps are independent of each other. The activation maps are generated when filters with fixed dimension, stride and padding are applied to the input. Stride refers to the amount by which a filter shifts during convolution and padding refers to filling 
the input volume by some number (mainly zeros or ones) around the border to preserve the spatial dimension. In the subsequent layers, the activation maps are given as inputs and the outputs are passed through a series of different types of layers to learn more complex features before being classified in the last layer. Other commonly used CNNs that are used for image recognition tasks include VGGNet [54], ResNet [24] and GoogLeNet [56].

\subsubsection{Evolution of CNNs}

Although the idea of convolutional neural networks (CNNs) dates back to 1980s, it gained popularity from 2012 by the dramatic success of AlexNet on image recognition task [34]. The network was relatively simple containing 5 convolutional layers, max-pooling layers, dropout layers, and 3 fully connected layers. This network achieved a record breaking performance on the ImageNet Dataset with a top-5 error rate of $15.4 \%$ and illustrated the benefits of techniques such as data augmentation and drop out for boosting the performance. After this huge breakthrough in the computer vision community, researchers began to use CNNs for a whole range of different applications and deeper networks came into the spotlight when VGGNet was proposed by Simonyan and Zisserman [54]. The network consists of 19 layers but it only uses $3 \times 3$ filters with stride and pad of 1, along with $2 \times 2$ max pooling layers with stride 2 . This network achieved good performance for both image classification and localization tasks. Their network was the first one to emphasize the importance of deeper networks. Since then, the CNNs started to become deeper. GoogLeNet [56] and ResNet [24] were proposed in 2015 that had about 100 and 152 layers respectively. In parallel, R-CNN [20] and their extensions- Fast RCNN [19] and Faster R-CNN [46], Generative Adversarial Networks [21], Spatial Transformer Networks [29] etc. became widely successful for a wide variety of other applications [3].

\subsection{Recurrent Neural Network (RNN)}

A recurrent neural network is a neural network which is widely used for sequential problems such as speech recognition and image captioning. RNNs have internal gates which allow them to capture and store long-term dependencies. An RNN en- 
codes temporal dependencies into the the network and computes the hidden state values based on the current input as well as the previous step hidden value. Figure 3.2 shows an example of the basic structure of an RNN. It shows the loop structure of an RNN and how it unrolls with time to form a chain-like structure where each unit $A$ is identical and is connected to the next one thus passing information. However, in practice RNNs suffer from vanishing or exploding gradient problems. Similar to traditional neural networks, RNNs are also associated with some nonlinear activation functions such as tanh that have gradients in the range $[-1,1]$ and during back propagation the gradients are being computed to learn the weights of the network. If the chain structure grows, the gradient becomes very small when computed with chain rule and multiplied with small numbers. The exploding gradient problem occurs when, due to similar reasons, the gradient grows exponentially. These problems prevent RNNs from learning temporal dependencies effectively.

In order to solve these problems, Long Short Term Memory (LSTM) networks were proposed by Hochreiter and Schmidhuber [26] that have special memory blocks to store or forget information which is not modified during the learning process allowing it to remember values for a long time. Figure 3.3 shows the gates that are present inside a standard LSTM unit, an input block and how they are operated. There are usually three types of gates inside an LSTM - the input gate controls the flow of information inside the memory, the output gate controls the information flow from the memory to the network and the forget gate controls which information to drop or keep in the memory. There are four different weight matrices for each of these gates that control the gate operations and the weights are learned during training the network. Figure 3.3 shows that all the gates are controlled by sigmoid functions and the output is connected back to the gates and the input block in a recurrent manner. There are different variations and extensions of LSTMs and for our work we have used Bidirectional LSTM [50] which is described in Chapter 4. 


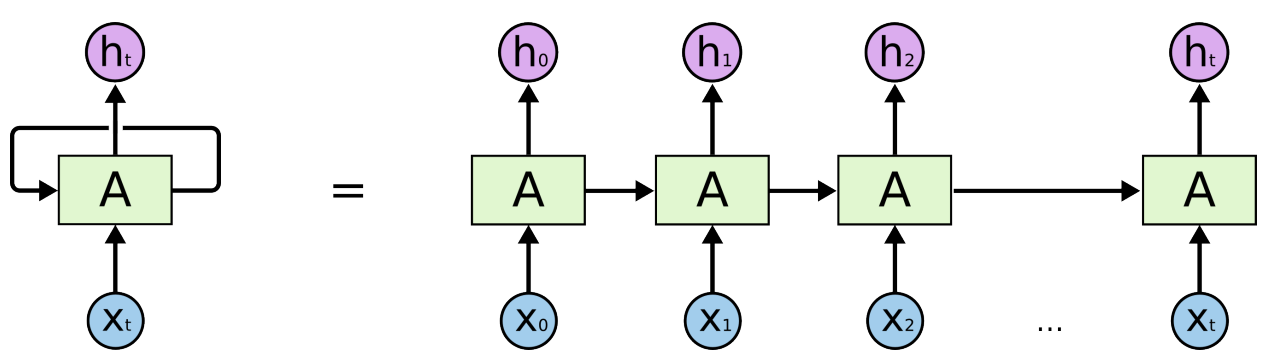

Figure 3.2: Block diagram of a simple RNN that unfolds with time forming a chain structure. A refers to one unit of the RNN, $x$ and $h$ refer to the input and the output at a given time step. The hidden state values of one unit are passed to the next one sequentially and are represented by the arrow. Source: [5]
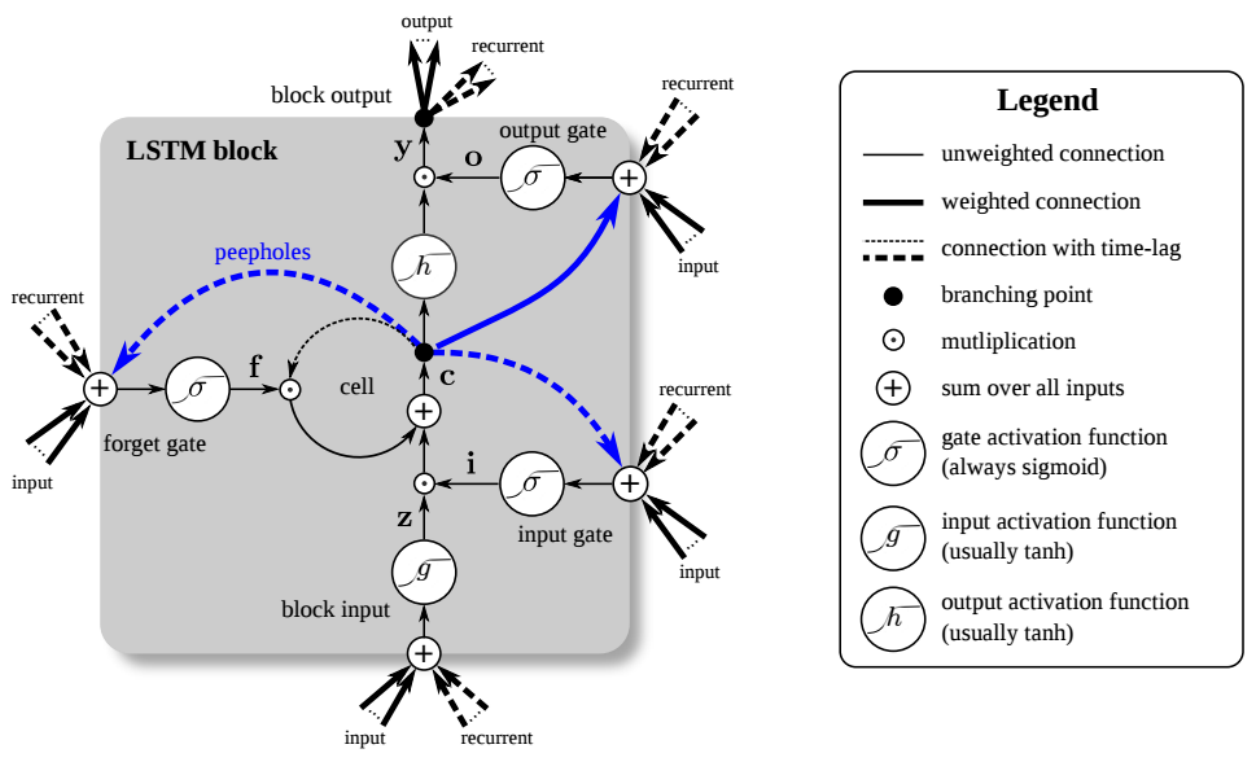

Figure 3.3: Building Block of an LSTM unit. Each LSTM unit has 3 gatesinput, output and forget, the input block and the output block. There are different types of weights associated with each component of the unit which are learned during training the network. The gates are usually controlled by sigmoid functions. Source: [4] 


\section{Chapter 4}

\section{Our Method}

\section{Method overview}

The input to our model is a sequence of images as well as the player bounding boxes and the homography matrix in each image. The bounding boxes of players can be in any order as our method does not require player trajectories. The output of the model is a group activity label for the entire sequence.

Our method has three stages: feature representation, feature aggregation and events prediction. In the feature representation and aggregation, we extract and aggregate different types of features that represent different aspects of the game. In the events prediction, we use a bidirectional LSTM model to classify the sequence of events. Our main efforts are on integrating different types of features to improve the classification accuracy.

\subsection{Individual Attributes}

We use appearance features to model individual players. The appearance feature is extracted by the $f c 7$ layer of AlexNet [34] using the sub-image of a player. We choose to use the pre-trained AlexNet (on ImageNet object recognition task) because it has been successfully used in various computer vision tasks [17, 28] and we do not have any player-level annotations such as individual actions. The output of the $\mathrm{CNN}$ represents the appearance information of an individual player.

Interaction among the individuals is essential to determine group activities. We 
use max pooling features of individual players in a particular frame to incorporate player interactions. Max pooling is a widely accepted technique that proved its effectiveness on a wide range of applications [28] [66]. We started by max pooling across all the players regardless of which team they are in. However this makes little sense because offending and defending players or players in different teams certainly behave and interact differently in any given sequence. This encouraged us to pool across the players by teams. Since we did not have any annotation to distinguish between the teams, we applied a segmentation and thresholding approach followed by max pooling and concatenation as shown in Figure 1.5 .

For the segmentation task, we adopted k-means clustering algorithm which segments an image by color. The value of $\mathrm{k}$ is 2 which refers to the number of clusters we want. In our case, one cluster refers to the player and the other cluster refers to the background. The input to the model is a player bounding box and the segmentation uses the jersey color of a particular player to separate him from the background. Since different games have different color jersey worn by the players, we manually set a threshold for each game to classify a player into one of the two teams. For example, for black and white jersey colors, if the most frequent pixel value exceeded 70, it was usually the white team. This simple thresholding approach gave satisfactory results as it did not matter for our task whether the color was correctly classified unless the players of the same team were correctly grouped together.

\subsection{Contextual Information from the Image}

We use deep features from a whole image to model the context information. In each frame, we use the $f c 7$ activation in fine-tuned AlexNet as the representation of the context. We have only fine-tuned the last two fully connected layers on our datasets and used pre-trained weights for the other layers. The intuition for adding this context is that some events can only be determined if we know the scene information. For example, if we consider the events dump in and dump out in ice hockey, they are almost the same except the fact that they occur at different zones. 


\subsection{Homography for the Spatial Feature Representation}

The homography matrix is a 3 by 3 matrix that can be applied to the input image pixels to get its warped version. In other words, if two images are taken from the same camera but have different viewpoints, we can compute the homography matrix to find the feature correspondences between the images. Some widely used applications of homography estimation include camera calibration, 3D reconstruction, stereo vision, scene understanding, camera motion, 3D modeling of objects, image registration and rectification. The homography matrix can be computed for both static and moving cameras and there is a wide range of literature proposed by researchers for computing the matrix [57] [23] [11] [18].

We took advantage of this homography matrix to incorporate the spatial arrangement of the players that in turn adds context to our knowledge domain. As mentioned earlier some events can only be distinguished in terms of the player location on the rink i.e. the zone information. Consider Figure 1.2 where the relative player arrangement is very similar for two different events: dump in and dump out. Thus looking at individual players or the relative positions of the players does not help us in identifying the events. However if we look at the spatial arrangement of the players with respect to each other as well as with respect to the rink positions, it is easier to distinguish those events. Moreover humans look at the whole image to identify certain events rather than looking at only a fraction of players. Keeping in mind that the camera is continuously moving, it is essential that we project the image coordinates to either template or world coordinate system. Homography matrix allows us to do this conversion easily. Thus given a homography matrix, our goal is to model the spatial player information that can be used as context features for our deep network.

\subsubsection{Computing Features from the Homography Matrix}

At first we computed the template coordinates of each player for each frame using Equation 4.1 where pit is the $i^{\text {th }}$ player in frame $\mathrm{t}, \mathrm{H}$ is the homography matrix, $\mathrm{T}$ is the template point and $\mathrm{I}$ is the image point. The image point corresponds to the bottom center of player bounding box. The world coordinate is just a linear translation and scaling of the template coordinates. So in our case it did not matter 


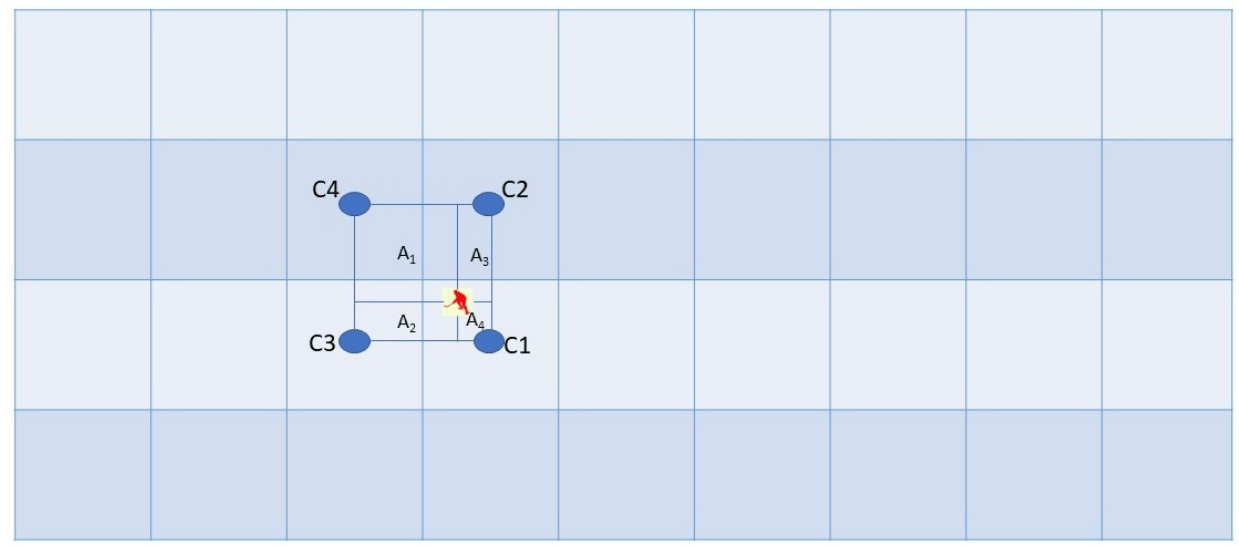

Figure 4.1: Weight computation method for a player and his associated centers.

whether we used template or world coordinate system. For visual convenience, we have used template coordinates. The template dimension of the ice hockey rink is $640 * 1440$. In order to keep the same aspect ratio, we divided the template image into $4 * 9$ bins. This gives us 36 bin centers in total. For each $p_{i}$ we have computed which 4 bin centers are its neighbors and what is the weight associated with each center with respect to $p_{i}$. The neighbors of $p_{i}$ refer to the bin centers that form a square around $p_{i}$. Equation 4.2 shows the weight computation method for center $\mathrm{c}$ at time t. The weights are summed over the total number of players, $n$ associated with c. Figure 4.1 shows how we have computed the weights for a center $c_{i}$ with respect to a particular player. At first, we find the nearest centers around the player such that they form a square. Then we divide the square into smaller rectangles from the player to the center locations. Next, we measure the area of each smaller rectangles. Since the weights should be higher for centers that are closer to the player than the centers that are further, we swap the areas as shown in the figure. For example, the weight for center $\mathrm{C} 1$ is equal to the area $\mathrm{A} 1$. For centers that do not have an associated player, the weights are 0 . The closer the center is from pi, higher the weight is for that center associated with pi. The weights for each square is normalized to sum to 1 . Thus the sum over all the centers is the number of players on the particular frame. Note that since there are fewer players than the total number of bins and players are usually clustered in a specific location 
of the rink, we get a very sparse matrix of weights. The next step is to combine the weight matrices with the deep features. This process is not very straightforward because the weights computed from the homography are hand-crafted features. We constructed our own late fusion model to fuse these features together.

$$
\begin{gathered}
T_{p i t}=h_{p i t} * I_{p i t} \\
W_{c t}=\sum_{p=1}^{n} w e i g h t(p)
\end{gathered}
$$

\subsection{Fusion Model for Combining Homography Features and Frame Features}

Inspired by Karpathy et al. [31], we have used a late fusion approach to incorporate the contextual cues. We have added fully connected layers to reduce all the feature vectors to similar dimension and then did late fusion on top. The model architecture is shown in figure 1.4. We first fine-tuned AlexNet so that the output from the $f c 7$ layer is reduced to 500 dimensions. The homography features were also passed through an $f c$ layer to produce a feature vector of 500 dimensions. The bounding box features are reduced to 1000 dimensions. These feature vectors were then concatenated and normalized before passing through two more $f c$ layers. The dimensions of the feature vectors and the choice of different layers were found empirically.

\subsection{Temporal Model}

We use a bidirectional Long Short Term Memory (BLSTM) network [50] to model the temporal information of the images. In every timestep $t$, the basic LSTM includes a hidden unit $h_{t}$, an input gate $i_{t}$, forget gate $f_{t}$, output gate $o_{t}$, input modulation gate $g_{t}$ and memory cell $c_{t}$. The LSTM formulation can be represented as the following equations: 


$$
\begin{array}{r}
i_{t}=\sigma\left(W_{x i} x_{t}+W_{h i} h_{t-1}+b_{i}\right) \\
f_{t}=\sigma\left(W_{x f} x_{t}+W_{h f} h_{t-1}+b_{f}\right) \\
o_{t}=\sigma\left(W_{x o} x_{t}+W_{h o} h_{t-1}+b_{o}\right) \\
g_{t}=\phi\left(W_{x c} x_{t}+W_{h c} h_{t-1}+b_{c}\right) \\
c_{t}=f_{t} \odot c_{t-1}+i_{t} \odot g_{t} \\
h_{t}=o_{t} \odot \phi\left(c_{t}\right)
\end{array}
$$

where $W$ terms denote weight matrices (e.g., $W_{x} i$ is the matrix of weights from the input to the input gate), $b$ terms are bias vectors. $\sigma$ is the logistic sigmoid function, $\phi$ is the tanh function, $\odot$ is the element-wise product. The BLSTM differs from LSTM by using two independent forward and backward LSTM layers. In both the forward and the backward passes, the input sequence and the ouput layer values are fed to the two hidden layers in the opposite directions. We have also tried more advanced LSTMs such as the LSTM with peephole connections [48]. There is no performance gain compared with the basic BLSTM model.

In this representation, the group dynamics is evolving over time and the event that occurs at a frame can be determined based on the hidden states computation from the preceding and the future time steps as well as the current input $x_{t}$. 


\section{Chapter 5}

\section{Experiments}

We have conducted experiments on two challenging sports datasets: an Ice Hockey Dataset provided by SPORTLOGiQ (http://sportlogiq.com/) and a publicly available Volleyball Dataset [28]. Our model can be divided into three distinct phases: feature extraction, feature aggregation and, learning and inference. We have used Matlab and TensorFlow [6] for all our experiments.

\subsection{Evaluation Metric}

We have reported the qualitative and quantitative results for both the datasets. The quantitative measure includes prediction accuracy with some tolerance, recall and precision per class and the confusion matrix. The prediction accuracy is a single value measurement for prediction evaluation. The output of all our temporal baselines and the final model is a probability distribution across all the classes. The general convention is to select the class having maximum probability as the predicted class. This is known as selecting the top-1 value. If the classes having the highest as well as the second highest probability are matched with the target class, we can call it top- 2 prediction. In our case, we have found that there is a huge performance gain if we choose the top- 2 values. However, we only have a very small number of target classes so top- 2 would not be a very reliable measurement for the performance evaluation of our model. We did a little analysis of the probabilities the model predicts and found that in most cases the model is highly confident 
about two predictions with a little probability difference between them and rest of the probabilities are very small numbers. This observation was particularly true for the minority classes that had very few training samples. This motivated us to pick top-2 predictions with a small tolerance value. Thus if the difference between the top-2 predictions is withing some tolerance value and if either of them matches the true class label, we consider it to be an accurate prediction. This is done because the Ice Hockey Dataset is highly imbalanced and in many confusing cases, the model predicts two classes with a very high probability. In those cases, the highest predicted probability is very close to the second highest probability. The tolerance value is set very low to ensure that we consider only those cases where the model is very confident about any two classes. This also eliminates cases where the second prediction has very low probability. For both the datasets, we have reported the accuracies for tolerance 0.0 and 0.15 for a fair comparison with the previous work. For the non-temporal baselines where we have used a SVM classifier, the tolerance is 0.0 , i.e. a prediction is correct only if the predicted class matches the true class because the output of the SVM is binary. The confusion matrix helps to visualize the per class recall and precision. This metric is particularly helpful for the Ice Hockey Dataset because the samples per class is highly imbalanced. If the model always predicts the majority class, the overall accuracy may be very high but the result would not reflect the true performance of the system. However with the help of the confusion matrix, it is easy to understand how the model performs with respect to each class. In our confusion matrix, the rightmost column shows the precision per class whereas the bottom row shows the recall per class in percentage. In general, recall measures the correctness of a model and precision measures the exactness. A high recall and precision is desired for the evaluation of any model and often it is a more reliable metric than the overall accuracy. In order to visualize the convergence of the network, we have also included the training cost and accuracy curves.

\subsection{Baselines}

We have considered the following baseline models for the evaluation of the datasets:

1. Frame-level Classification with CNN (M1): This baseline extracts frame 
level features from target frames and classifies the event for the target frames using a Support Vector Machine (SVM).

2. Person-level Classification with CNN (M2): This baseline first extracts player level features from target frames. Then it max pools across the teams and classifies events for target frames using a SVM.

3. Frame-level Temporal Model (M3): This is an extension of the first baseline (M1). Instead of using the target frames and SVM classification, this method feeds the frame level features from the whole sequence into an LSTM to classify events for the whole sequence.

4. Person-level Temporal Model (M4): This is an extension of the second baseline (M2). It feeds the player level features from a sequence of images to an LSTM to classify events for the whole sequence.

5. Frame-level Classification with fine-tuned CNN (M5): This baseline is similar to M1 but we fine-tuned AlexNet using the target frame events and classified using softmax.

6. Frame-level Temporal Model (M6): This baseline is same as M3 but it uses fine-tuned AlexNet features rather than pre-trained features.

7. Frame and Person Fusion Model (M7): This baseline fine-tunes AlexNet and adds a late fusion on top of it to combine the person level and the frame level features. The classification is done using softmax.

8. Frame and Person Fusion Model with LSTM (M8): This baseline is a temporal extension of M7. The features from the fusion model are passed into an LSTM for temporal classification.

9. Our non-temporal method (M9): This baseline fine-tunes AlexNet and adds a late fusion on top of it to combine the person level, the frame level and the homography features. It does not encode any temporal information. The prediction is done using softmax. 
We also compare our method with the C3D [58] network. The C3D network is pre-trained on the UCF101 action recognition dataset [55] and fine-tuned on our dataset. For the Volleyball Dataset, we have additionally reported the previous state-of-the-art results.

\subsection{Implementation Details}

We extracted deep features from the player bounding boxes in Matlab using an AlextNet pre-trained on the ImageNet for object recognition task. We also used Matlab for extracting the homography features from the homography matrices. All other experiments were conducted using TensorFlow [6] framework. For the image feature extraction, we used the AlexNet fine-tuned on our datasets if not specified otherwise. For the prediction model, we used either a support vector machine (SVM) or a softmax classifier. Our LSTM network consists of 28 hidden nodes, 500 input features and optimizes weighted softmax cross entropy loss function. The weight for each class is inversely proportional to the frequency of the samples per class in the dataset normalized to sum to 1 . We used a learning rate of 0.0000005 , batch size of 256, 50\% dropout, batch-normalization and Adam optimizer [32]. Only the predictions at the states that correspond to the target frames are used as the classification probabilities and the loss is computed only on the target frames during training. Figures 5.2 and 5.3 show the training loss and accuracy changes respectively as a function of time for the Ice Hockey Dataset.

For the Volleyball Dataset, we used a batch size of 500. The dataset was not imbalanced like the Ice Hockey Dataset so we used a regular softmax cross entropy loss function instead of the weighted loss. The other hyper-parameters were same as ice hockey. Figures 5.6 and 5.7 show the training loss and accuracy changes respectively as a function of time for the Volleyball Dataset.

\subsection{Ice Hockey Dataset}

This dataset consists of National Hockey League (NHL) videos and was obtained from SPORTLOGiQ (http://sportlogiq.com/). We used part of this dataset and considered five puck possession events. Table 5.1 shows the descriptions of the events and corresponding number of instances in the dataset. It clearly shows that some of 
the events occur very rarely such as dump in. We addressed this imbalance by minimizing weighted softmax cross entropy loss. We randomly used 3966 events for training and 270 events for testing. The dataset has the annotated frame numbers where an event occurred and we used the preceding and future frames for our temporal classification. All the events are considered to be independent of each other and were trained as individual short clips of events, which is a standard protocol used for activity recognition tasks [28][58].

\subsubsection{Event Annotation}

In this dataset, an example contains a target frame which is associated with an event label. The target frame is generally a frame that marks the beginning of an event. We do not have any annotation for the duration of an event. To solve this problem, one naive approach would be to mark all the frames up to the next event as the current event. However, in the Ice Hockey Dataset, we have found that although sometimes the naive approach could make sense, in many cases the intermediate frames either belonged to the negative example class or the next event. By the negative example class we refer to the frames that are not associated with any event. As a result, we could not make that assumption. Rather we fixed the maximum length of a sequence to be 24 . This included 18 frames before and 5 frames after the target frame. If two events were less than 18 frames apart, we marked all the preceding and succeeding frames as belonging to the target frame. The maximum length of a sequence was found empirically.

\subsubsection{Tracking Data}

The dataset have annotations for the player bounding boxes in each frame. The bounding boxes are annotated by the $x, y$, width, height format to mark the location of each player in the pixel coordinate system. However, the referee bounding box is also annotated in most of the frames and there is no annotation to distinguish the referee from other players. As a result the features from the referee are also included when we extracted the deep features from the player bounding boxes. Some of the bounding box annotations are very noisy as well especially in cases where there is occlusion or the players are very close to each other. In these cases, 

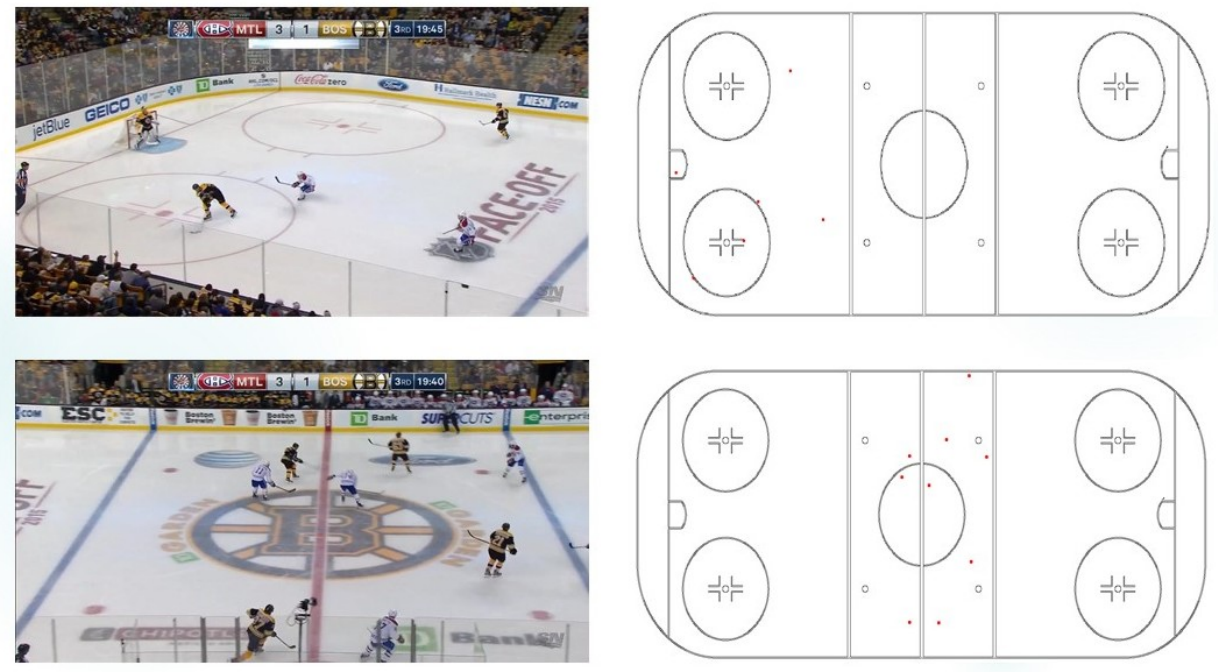

Figure 5.1: The transformation from the image to the template coordinate system by the homography matrix.

there is a big bounding box that surrounds multiple players. In our model, we have only used the bounding box coordinates of all the annotated instances to crop the players out of the frame and extract deep features to incorporate their appearance information. We have not used any other tracking information.

\subsubsection{Homography Transformation}

The dataset contains the homography matrix for each frame that projects the video frame to the template. We have used these matrices to calculate the homography features which is described in Chapter 4. Figure 5.1 shows some examples of this transformation. The players in each frame are denoted by the small red dots in the template image.

\subsubsection{Quantitative Results}

Table 5.3 shows the classification accuracy of our models and the baselines. Our non-temporal method outperforms all the baselines except M5 and M7 where the tolerance is 0.0 and only M7 where the tolerance is 0.15 . The temporal extension also produces competitive results. The accuracies for both tolerance 0.0 and 


\begin{tabular}{|ccc|}
\hline Event & \multicolumn{1}{c|}{ Description } & Examples \\
\hline $\begin{array}{c}\text { Loose puck recovery } \\
\text { (LPR) }\end{array}$ & $\begin{array}{c}\text { The player recovered } \\
\text { the puck as it was out of } \\
\text { possession of any player }\end{array}$ & 1,412 \\
\hline Pass & $\begin{array}{c}\text { The player attempts a } \\
\text { pass to a teammate }\end{array}$ & 1,688 \\
\hline Shot & A player shoots on goal & 346 \\
\hline Dump in & $\begin{array}{l}\text { When a player sends the } \\
\text { puck into the offensive } \\
\text { zone }\end{array}$ & 319 \\
\hline Dump out & $\begin{array}{l}\text { When a defending } \\
\text { player dumps the puck } \\
\text { up the boards without } \\
\text { targeting a teammate for } \\
\text { a pass }\end{array}$ \\
\hline
\end{tabular}

Table 5.1: Event descriptions and corresponding number of training examples in the Ice Hockey Dataset.

\begin{tabular}{|c|c|}
\hline Baseline & Descriptions \\
\hline M1 & Frame features from pre-trained AlexNet classified with SVM \\
M2 & Person features from pre-trained AlexNet classified with SVM \\
M3 & Temporal extension of M1 classified with softmax \\
M4 & Temporal extension of M2 classified with softmax \\
M5 & Frame features from fine-tuned AlexNet classified with softmax \\
M6 & Temporal extension of M5 classified with softmax \\
M7 & Fusion of frame and person features classified with softmax \\
M8 & Temporal extension of M7 classified with softmax \\
\hline
\end{tabular}

Table 5.2: The descriptions of the baselines. 


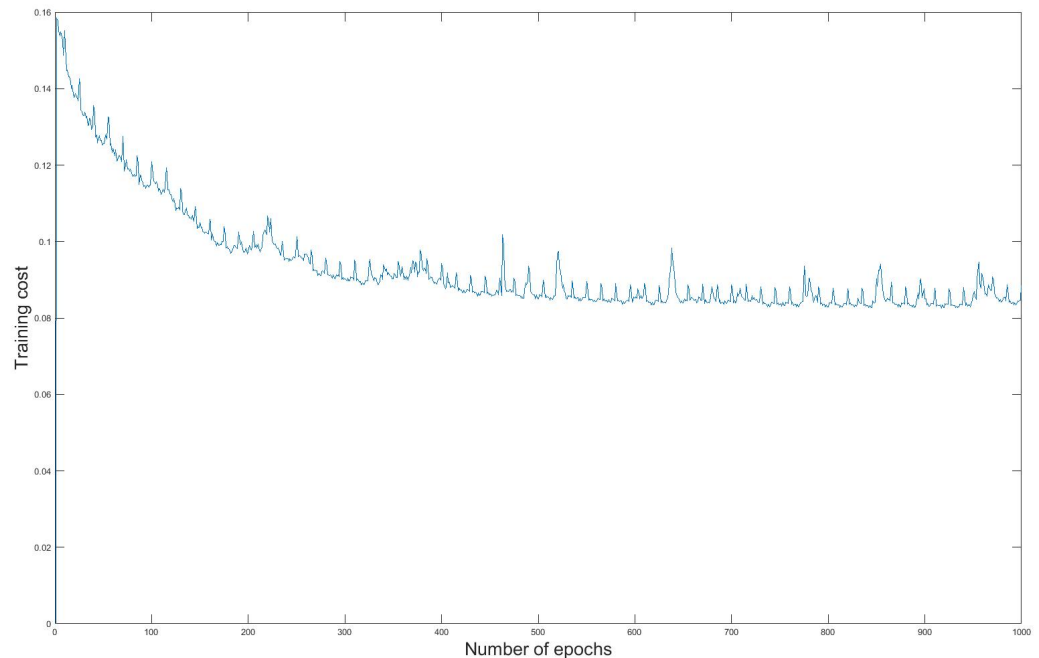

Figure 5.2: Training loss over time for the Ice Hockey Dataset.

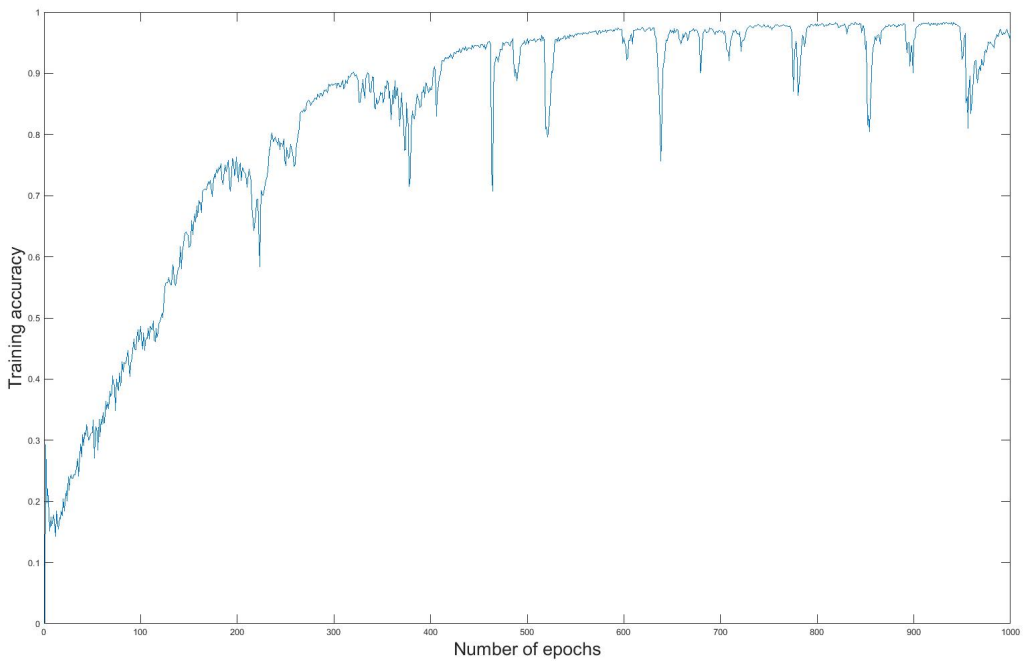

Figure 5.3: Training accuracy over time for the Ice Hockey Dataset. 


\begin{tabular}{|c|c|c|c|c|c|c|}
\hline \multirow[b]{2}{*}{ LPR } & \multicolumn{6}{|c|}{ Confusion Matrix } \\
\hline & $\begin{array}{c}\mathbf{4 1} \\
15.2 \%\end{array}$ & $\begin{array}{c}32 \\
11.9 \%\end{array}$ & $\begin{array}{c}\mathbf{4} \\
1.5 \%\end{array}$ & $\begin{array}{c}\mathbf{5} \\
1.9 \%\end{array}$ & $\begin{array}{c}\mathbf{6} \\
2.2 \%\end{array}$ & $\begin{array}{l}46.6 \% \\
53.4 \%\end{array}$ \\
\hline Pass & $\begin{array}{c}\mathbf{5 1} \\
18.9 \%\end{array}$ & $\begin{array}{c}73 \\
27.0 \%\end{array}$ & $\begin{array}{c}\mathbf{6} \\
2.2 \%\end{array}$ & $\begin{array}{c}11 \\
4.1 \%\end{array}$ & $\begin{array}{c}\mathbf{6} \\
2.2 \%\end{array}$ & $\begin{array}{l}49.7 \% \\
50.3 \%\end{array}$ \\
\hline Shot & $\begin{array}{c}\mathbf{4} \\
1.5 \%\end{array}$ & $\begin{array}{c}\mathbf{6} \\
2.2 \%\end{array}$ & $\begin{array}{c}3 \\
1.1 \%\end{array}$ & $\begin{array}{c}1 \\
0.4 \%\end{array}$ & $\begin{array}{c}1 \\
0.4 \%\end{array}$ & $\begin{array}{l}20.0 \% \\
80.0 \%\end{array}$ \\
\hline Dump in & $\begin{array}{c}\mathbf{5} \\
1.9 \%\end{array}$ & $\begin{array}{c}3 \\
1.1 \%\end{array}$ & $\begin{array}{c}\mathbf{0} \\
0.0 \%\end{array}$ & $\begin{array}{c}1 \\
0.4 \%\end{array}$ & $\begin{array}{c}1 \\
0.4 \%\end{array}$ & $\begin{array}{l}10.0 \% \\
90.0 \%\end{array}$ \\
\hline ump out & $\begin{array}{c}\mathbf{4} \\
1.5 \%\end{array}$ & $\begin{array}{c}\mathbf{4} \\
1.5 \%\end{array}$ & $\begin{array}{c}\mathbf{0} \\
0.0 \%\end{array}$ & $\begin{array}{c}\mathbf{0} \\
0.0 \%\end{array}$ & $\begin{array}{c}\mathbf{2} \\
0.7 \%\end{array}$ & $\begin{array}{l}20.0 \% \\
80.0 \%\end{array}$ \\
\hline ALL & $\begin{array}{l}39.0 \% \\
61.0 \%\end{array}$ & $\begin{array}{l}61.9 \% \\
38.1 \%\end{array}$ & $\begin{array}{l}23.1 \% \\
76.9 \%\end{array}$ & $\begin{array}{c}5.6 \% \\
94.4 \%\end{array}$ & $\begin{array}{l}12.5 \% \\
87.5 \%\end{array}$ & $\begin{array}{l}44.4 \% \\
55.6 \%\end{array}$ \\
\hline & LPR & Pass & Shot & Dump in & Dump out & ALL \\
\hline
\end{tabular}

Figure 5.4: Confusion matrix for top- 1 event prediction with a tolerance of 0.0 on the Ice Hockey Dataset. The rightmost column shows the precision and the bottom row shows the recall per class in percentages. The green values represent the percentages of the correct predictions by the model whereas the red values show the percentages of the incorrect predictions. For example, recall for $l p r$ is $39 \%$ and the its precision is $46.6 \%$ which means out of all the $l p r$ events, $39 \%$ were correctly classified and out of all the lpr predictions made by the model, $46.6 \%$ were correct. The blue rectangle in the bottom right corresponds to the overall accuracy. 


\begin{tabular}{|c|c|c|c|c|c|c|}
\hline \multirow[b]{2}{*}{ LPR } & \multicolumn{6}{|c|}{ Confusion Matrix } \\
\hline & $\begin{array}{c}78 \\
28.9 \%\end{array}$ & $\begin{array}{c}\mathbf{9} \\
3.3 \%\end{array}$ & $\begin{array}{c}3 \\
1.1 \%\end{array}$ & $\begin{array}{c}\mathbf{4} \\
1.5 \%\end{array}$ & $\begin{array}{c}\mathbf{6} \\
2.2 \%\end{array}$ & $\begin{array}{l}78.0 \% \\
22.0 \%\end{array}$ \\
\hline Pass & $\begin{array}{c}20 \\
7.4 \%\end{array}$ & $\begin{array}{c}100 \\
37.0 \%\end{array}$ & $\begin{array}{c}\mathbf{5} \\
1.9 \%\end{array}$ & $\begin{array}{c}10 \\
3.7 \%\end{array}$ & $\begin{array}{c}\mathbf{5} \\
1.9 \%\end{array}$ & $\begin{array}{l}71.4 \% \\
28.6 \%\end{array}$ \\
\hline Shot & $\begin{array}{c}3 \\
1.1 \%\end{array}$ & $\begin{array}{c}\mathbf{4} \\
1.5 \%\end{array}$ & $\begin{array}{c}\mathbf{4} \\
1.5 \%\end{array}$ & $\begin{array}{c}1 \\
0.4 \%\end{array}$ & $\begin{array}{c}\mathbf{0} \\
0.0 \%\end{array}$ & $\begin{array}{l}33.3 \% \\
66.7 \%\end{array}$ \\
\hline Dump in & $\begin{array}{c}\mathbf{2} \\
0.7 \%\end{array}$ & $\begin{array}{c}3 \\
1.1 \%\end{array}$ & $\begin{array}{c}1 \\
0.4 \%\end{array}$ & $\begin{array}{c}3 \\
1.1 \%\end{array}$ & $\begin{array}{c}\mathbf{0} \\
0.0 \%\end{array}$ & $\begin{array}{l}33.3 \% \\
66.7 \%\end{array}$ \\
\hline Dump out & $\begin{array}{c}\mathbf{2} \\
0.7 \%\end{array}$ & $\begin{array}{c}\mathbf{2} \\
0.7 \%\end{array}$ & $\begin{array}{c}\mathbf{0} \\
0.0 \%\end{array}$ & $\begin{array}{c}\mathbf{0} \\
0.0 \%\end{array}$ & $\begin{array}{c}\mathbf{5} \\
1.9 \%\end{array}$ & $\begin{array}{l}55.6 \% \\
44.4 \%\end{array}$ \\
\hline ALL & $\begin{array}{l}74.3 \% \\
25.7 \%\end{array}$ & $\begin{array}{l}84.7 \% \\
15.3 \%\end{array}$ & $\begin{array}{l}30.8 \% \\
69.2 \%\end{array}$ & $\begin{array}{l}16.7 \% \\
83.3 \%\end{array}$ & $\begin{array}{l}31.2 \% \\
68.8 \%\end{array}$ & $\begin{array}{l}70.4 \% \\
29.6 \%\end{array}$ \\
\hline & LPR & Pass & Shot & Dump in & Dump out & ALL \\
\hline
\end{tabular}

Figure 5.5: Confusion matrix for top- 2 event prediction with a tolerance of 0.15 on the Ice Hockey Dataset. The rightmost column shows the precision and the bottom row shows the recall per class in percentages. The green values represent the percentages of the correct predictions by the model whereas the red values show the percentages of the incorrect predictions. For example, recall for $l p r$ is $74.3 \%$ and the its precision is $78 \%$ which means out of all the $l p r$ events, $74.3 \%$ were correctly classified and out of all the $l p r$ predictions made by the model, $78 \%$ were correct. The blue rectangle in the bottom right corresponds to the overall accuracy. 


\begin{tabular}{|c|c|c|c|}
\hline Method & Fine-tuning & Acc(\%)Tol=0.0 & Acc(\%)Tol=0.15 \\
\hline M1 & w/o & 32.4 & - \\
M2 & w/o & 30.5 & - \\
M3 & w/o & 40.1 & 58 \\
M4 & w/o & 35.4 & 65.6 \\
M5 & w & 46.9 & 75.8 \\
M6 & w & 40.4 & 60 \\
M7 & w & 48.4 & 79.7 \\
M8 & w & 40.0 & 62.6 \\
C3D [58] & w & 44.0 & - \\
Our non-temporal method & w & 45.7 & 77.3 \\
\hline Our temporal method & w & 44.4 & 70.4 \\
\hline
\end{tabular}

Table 5.3: Performance of our model on Ice Hockey Dataset compared to the baselines. In the fine-tuning column, w/o and w represent without and with fine-tuning, respectively.

\begin{tabular}{|ccccc|}
\hline Event & $\begin{array}{c}\text { Recall (\%) } \\
\text { Tol=0.0 }\end{array}$ & $\begin{array}{c}\text { Precision (\%) } \\
\text { Tol=0.0 }\end{array}$ & $\begin{array}{c}\text { Recall (\%) } \\
\text { Tol=0.15 }\end{array}$ & $\begin{array}{c}\text { Precision (\%) } \\
\text { Tol=0.15 }\end{array}$ \\
\hline LPR & 39 & 46.6 & 74.3 & 78 \\
Pass & 61.9 & 49.7 & 84.7 & 71.4 \\
Shot & 23.1 & 20 & 30.8 & 33.3 \\
Dump in & 5.6 & 10 & 16.7 & 33.3 \\
Dump out & 12.5 & 20 & 31.2 & 55.6 \\
\hline Average & 28.42 & 29.26 & 47.54 & 54.32 \\
\hline
\end{tabular}

Table 5.4: Per class recall and precision of our model on the Ice Hockey Dataset.

tolerance 0.15 show similar patterns.

If we compare the first six baselines with a tolerance of 0.0 , the frame level features work better than the player level features. For example, M1, M3 and M5 outperforms M2, M4 and M6. Adding frame level features is important because events such as dump in and dump out can possibly be distinguished only by zone information. Another observation is that fine-tuned frame features perform better than the pre-trained frame features because the AlexNet is pre-trained from 
an image recognition task in which sample images usually have an object in the middle of the frame. When we apply this pre-trained model to our problem, it is more suitable for extracting player level features as players are in the middle of the frame. On the other hand, a whole frame has multiple players and large areas of background, thus the pre-trained AlexNet becomes less suitable. That is why we have fine-tuned AlexNet for frame level classification using the target frame events. M5 and M6 show that the fine-tuned frame features outperform both M1 and M3. However, we had challenges to fine-tune bounding box features because we do not have person level annotations.

Another interesting observation is that although M3 and M4 perform better than M1 and M2 after encoding the temporal information, in all other cases the non-temporal versions perform significantly better than the temporal extensions. For example, M7 performs better than M8. When we dug deeper, we found that although the overall accuracy is higher for the non-temporal models, the model always predicted either lpr or pass events and never predicted the rest. But when we encode temporal knowledge, per class accuracy was much better. This indicates that the features evolve over the time as they approach the target events and it becomes easier for the model to predict the minority classes. When we provide only the target frames as inputs to our models, they find it difficult to separate the less frequent classes from the more frequent ones in the training set.

Next, we compare all the fusion models and C3D. Our non-temporal and temporal models perform better than M8 and C3D. The accuracy of M7 is the highest among all the baselines which fuses frame features with the player features. However, the confusion matrix showed that per class recall and precision was the best for the temporal model where we fused homography, player and frame features, although the overall accuracy is lower.

Figures 5.4 and 5.5 show the confusion matrices of the events. Figure 5.4 shows that the model performs quite well in classifying $l p r$ and pass but it seriously fails on the other three events. If we look at columns 3-5 and rows 1-2 of the matrix, it is clearly seen that these three events are mostly misclassified as lpr or pass. For example, 11 and 5 dump in events are misclassified as pass and lpr events respectively. This is because the training dataset is highly imbalanced and the less frequent events perform poorly compared to the dominant events. 
However, if we look at Figure 5.5 that considers the top-2 predictions with a very small probability difference, the overall performance gain is approximately $60 \%$ and the per class performance also improves significantly. Although the classification rate is still not very high, a comparison between the two confusion matrices shows that class imbalance is a major concern for this dataset which forces the model to always predict the majority classes although sometimes it is highly confident about other classes. The class imbalance is present because we considered complete games of the ice hockey videos rather than handpicking certain clips and, in any ice hockey game, some events will always occur more frequently compared to others. One solution could be to ignore the majority events in certain games and handpick the minority classes but that would be possible only if we have many available games in the dataset. Another way could be adding augmented clips which is not trivial like adding augmented images.

Table 5.4 shows the recall and precision values for each class. It is again seen that $l p r$ and pass have much higher figures compared to rest of the events. It is also seen that slightly increasing the tolerance makes a huge shift in performance for all the classes.

\subsubsection{Qualitative Results}

Figures 5.10, 5.11 and 5.12 show the qualitative results of our model for event prediction in Ice Hockey Dataset. Figure 5.10 shows the correct predictions with a tolerance of 0.0 , Figure 5.11 shows the wrong predictions by the model with tolerance 0.0 and the correction by the model with tolerance 0.15 with the change in probability between the two predictions and Figure 5.12 shows the failed predictions. Our model could successfully identify some challenging scenarios such as blurred image as seen in Figure 5.10. On the other side, Figure 5.11 shows that our model fails to predict the less frequent events with the highest confidence but the prediction was accurate for some difficult cases if we consider the top- 2 predictions. 


\begin{tabular}{|c|c|}
\hline Event & Description \\
\hline Set & $\begin{array}{r}\text { The setter, located in the center front, hits the ball high above the } \\
\text { net so that a spiker can spike it across }\end{array}$ \\
\hline Spike & $\begin{array}{r}\text { When an offensive player attacks the ball with a one-arm motion } \\
\text { done over the head, attempting to get a kill }\end{array}$ \\
\hline Pass & $\begin{array}{c}\text { Receiving a serve or the first contact of the ball with the intent to } \\
\text { control the ball to another player }\end{array}$ \\
\hline Winpoint & When a team scores \\
\hline
\end{tabular}

Table 5.5: Event descriptions in the Volleyball Dataset. In the original dataset and all our experiments, these events are further classified into right and left where right always refers to the team in the right side of the net and left refers to the team in the left side. For example, r-pass refers to the pass event occurring in the right side.

\subsection{Volleyball Dataset}

We have used the publicly available Volleyball Dataset provided by Ibraim et al. [28]. The dataset contains annotations for individual players as well as group activities of 4830 randomly picked frames of 55 different games from YouTube. We have only used the event annotations and the bounding boxes of the players in our experiments. We have used 5 frames before and 4 frames after the target frame for the temporal sequence. Since the homography matrix is not available for this dataset, we conducted our experiments without the homography features. Table 5.5 shows the event descriptions in the Volleyball Dataset.

\subsubsection{Quantitative Results}

Table 5.7 summarizes the accuracy of all the experiments on the Volleyball Dataset. For this dataset, we could only compare the baseline models and previous state-ofthe-arts because we did not have the homography matrices. Similar to ice hockey results, similar pattern in performance is observed for both tolerance 0.0 and 0.15 .

The model proposed by Bagautdinov et al. [8] outperforms all the baselines by a significant margin. On the other hand, M2 performs surprisingly better than most other baselines although it encodes only bounding box features and does not have any temporal knowledge. This shows the huge benefit of team pooling in volley- 


\begin{tabular}{|c|c|}
\hline Baseline & Descriptions \\
\hline M1 & Frame features from pre-trained AlexNet classified with SVM \\
M2 & Person features from pre-trained AlexNet classified with SVM \\
M3 & Temporal extension of M1 classified with softmax \\
M4 & Temporal extension of M2 classified with softmax \\
M5 & Frame features from fine-tuned AlexNet classified with softmax \\
M6 & Temporal extension of M5 classified with softmax \\
M7 & Fusion of frame and person features classified with softmax \\
M8 & Temporal extension of M7 classified with softmax \\
\hline
\end{tabular}

Table 5.6: The Descriptions of the baselines.

ball games mostly because the teams are well defined and separated in volleyball. Another interesting finding is fine-tuning with only the frame features gave a very low accuracy. But when we combined the fine-tuned frame features with the player features in M8, the accuracy was much higher.

Table 5.8 shows the per class recall and precision results when we applied M8 on the Volleyball Dataset. Unlike Ice Hockey Dataset, the Volleyball Dataset did not have any imbalance among the classes and the results show that the per class performance is also similar. Only for the event, $r$-winpoint, we found a low accuracy. However when we increased the tolerance value to 0.15 , the precision for this event jumped to the highest with a notable increase in recall compared to all other events. Analyzing the confusion matrix shows that most $r$-winpoints are confused with l-winpoints.

Figures 5.8 and 5.9 show the confusion matrices of our model on the Volleyball Dataset. There was a significant performance gain of $68 \%$ when we increased the tolerance value. The classification accuracy, recall and precision were highly balanced across all the events unlike the Ice Hockey Dataset.

\subsubsection{Qualitative Results}

Figure 5.13 shows the example images where our model predicted the correct events with a tolerance of 0.0. If we look at the bottom left image, it is seen that both the teams are wearing red and white jerseys and it is hard to distinguish 


\begin{tabular}{|cccc|}
\hline Method & Fine-tuning & Acc(\%)Tol=0.0 & Acc(\%)Tol=0.15 \\
\hline M1 & w/o & 37.7 & - \\
\hline M2 & w/o & 47.6 & - \\
\hline M3 & w/o & 38.1 & 60.1 \\
\hline M4 & w/o & 39 & 61.3 \\
\hline M5 & w & 37 & 58 \\
\hline M6 & w & 34 & 61.4 \\
\hline M7 & w & 35.6 & 57.2 \\
\hline M8 & w & 47.5 & 69.2 \\
\hline C3D [58] & w & 74 & - \\
\hline $\begin{array}{c}\text { Two-stage } \\
\text { Hierarchical } \\
\text { Model [28] }\end{array}$ & w & 51.1 & - \\
\hline $\begin{array}{c}\text { Social Scene } \\
\text { Understanding } \\
{[8]}\end{array}$ & & & - \\
\hline
\end{tabular}

Table 5.7: Performance of our model on Volleyball Dataset compared to the baselines and previous work. $\mathrm{w}$ and w/o refers to with and without finetuning respectively.

\begin{tabular}{|ccccc|}
\hline Event & $\begin{array}{c}\text { Recall (\%) } \\
\text { Tol=0.0 }\end{array}$ & $\begin{array}{c}\text { Precision (\%) } \\
\text { Tol=0.0 }\end{array}$ & $\begin{array}{c}\text { Recall (\%) } \\
\text { Tol=0.15 }\end{array}$ & $\begin{array}{c}\text { Precision (\%) } \\
\text { Tol=0.15 }\end{array}$ \\
\hline r-set & 41.7 & 51.3 & 59.9 & 70.9 \\
r-spike & 51.2 & 43.2 & 67.3 & 63.1 \\
r-pass & 51.5 & 46.1 & 70.6 & 63.7 \\
r-winpoint & 15.9 & 36.1 & 68.3 & 80 \\
l-winpoint & 53.1 & 46.4 & 69.8 & 72.8 \\
l-pass & 44.5 & 45 & 71.4 & 69.5 \\
l-spike & 59.3 & 52.5 & 77.4 & 70.3 \\
l-set & 48.8 & 51.6 & 68.1 & 73.4 \\
\hline Average & 45.75 & 46.525 & 69.1 & 70.46 \\
\hline
\end{tabular}

Table 5.8: Per class recall and precision of our model on the Volleyball Dataset. 


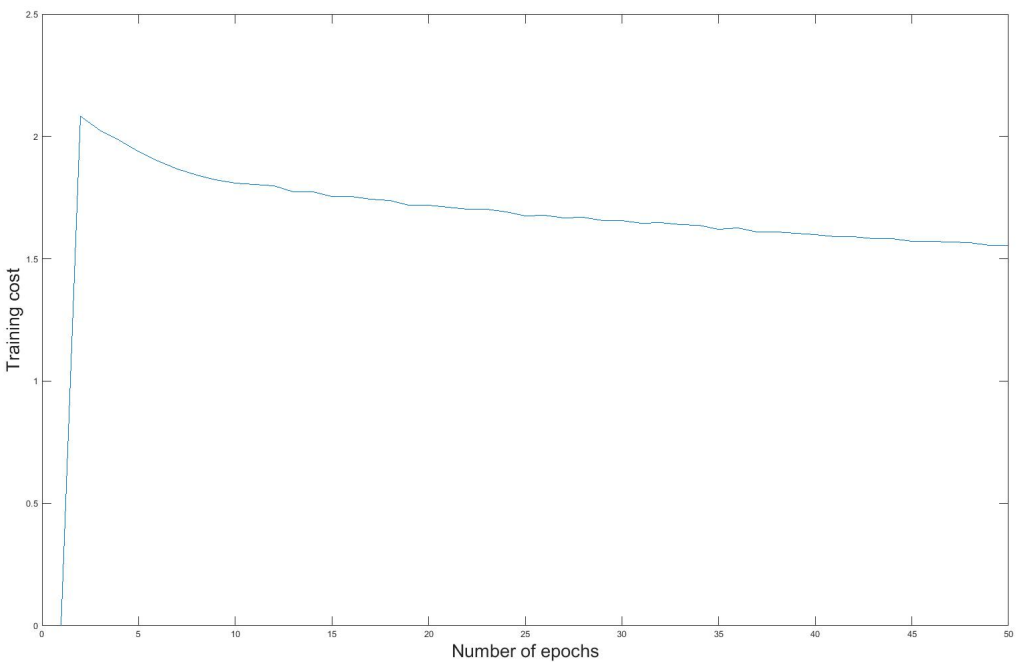

Figure 5.6: Training loss over time for the Volleyball Dataset.

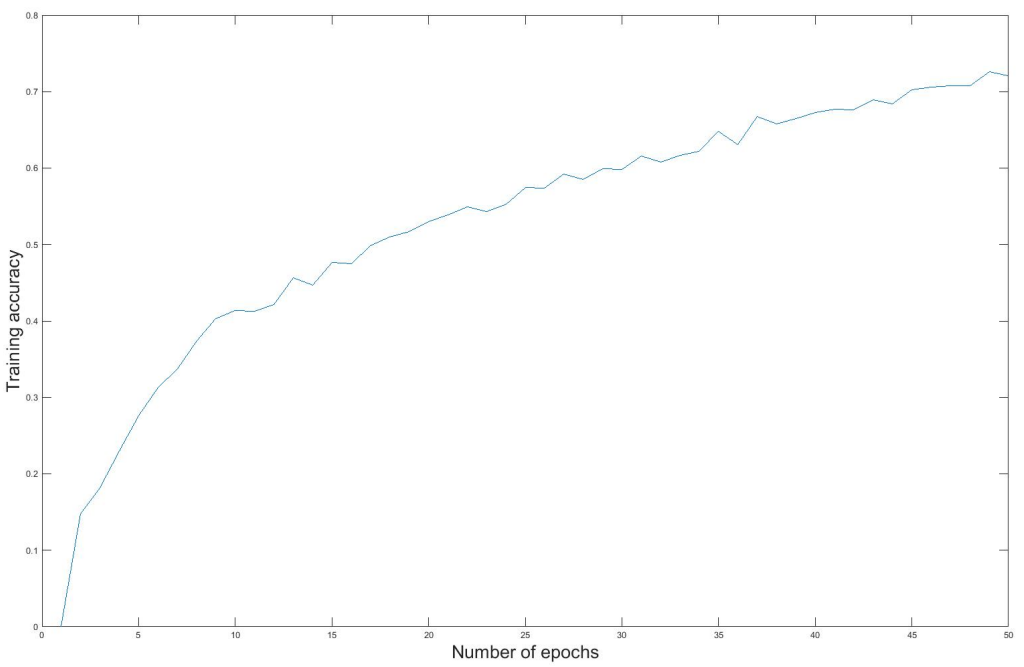

Figure 5.7: Training accuracy over time for the Volleyball Dataset. 


\begin{tabular}{|c|c|c|c|c|c|c|c|c|c|}
\hline & & & & & nfusion ima & atrix & & & \\
\hline r-set & $\begin{array}{c}78 \\
6.0 \%\end{array}$ & $\begin{array}{c}\mathbf{2 0} \\
1.5 \%\end{array}$ & $\begin{array}{c}19 \\
1.5 \%\end{array}$ & $\begin{array}{c}\mathbf{0} \\
0.0 \%\end{array}$ & $\begin{array}{c}3 \\
0.2 \%\end{array}$ & $\begin{array}{c}\mathbf{6} \\
0.5 \%\end{array}$ & $\begin{array}{c}7 \\
0.5 \%\end{array}$ & $\begin{array}{c}19 \\
1.5 \%\end{array}$ & $\begin{array}{l}51.3 \% \\
48.7 \%\end{array}$ \\
\hline r-spike & $\begin{array}{c}16 \\
1.2 \%\end{array}$ & $\begin{array}{c}86 \\
6.6 \%\end{array}$ & $\begin{array}{c}\mathbf{1 7} \\
1.3 \%\end{array}$ & $\begin{array}{c}\mathbf{6} \\
0.5 \%\end{array}$ & $\begin{array}{c}\mathbf{6} \\
0.5 \%\end{array}$ & $\begin{array}{c}43 \\
3.3 \%\end{array}$ & $\begin{array}{c}13 \\
1.0 \%\end{array}$ & $\begin{array}{c}12 \\
0.9 \%\end{array}$ & \\
\hline & $\begin{array}{c}42 \\
3.2 \%\end{array}$ & $\begin{array}{c}10 \\
0.8 \%\end{array}$ & $\begin{array}{c}105 \\
8.1 \%\end{array}$ & $\begin{array}{c}7 \\
0.5 \%\end{array}$ & $\begin{array}{c}15 \\
1.2 \%\end{array}$ & $\begin{array}{c}19 \\
1.5 \%\end{array}$ & $\begin{array}{c}19 \\
1.5 \%\end{array}$ & $\begin{array}{c}11 \\
0.8 \%\end{array}$ & \\
\hline & $\begin{array}{c}1 \\
0.1 \%\end{array}$ & $\begin{array}{c}\mathbf{2} \\
0.2 \%\end{array}$ & $\begin{array}{c}3 \\
0.2 \%\end{array}$ & $\begin{array}{c}13 \\
1.0 \% \\
\end{array}$ & $\begin{array}{c}\mathbf{8} \\
0.6 \%\end{array}$ & $\begin{array}{c}7 \\
0.5 \%\end{array}$ & $\begin{array}{c}\mathbf{1} \\
0.1 \%\end{array}$ & $\begin{array}{c}\mathbf{1} \\
0.1 \%\end{array}$ & $\begin{array}{l}36.10 \\
63.9^{\circ} \\
\end{array}$ \\
\hline & $\begin{array}{c}\mathbf{2} \\
0.2 \%\end{array}$ & $\begin{array}{c}\mathbf{5} \\
0.4 \%\end{array}$ & $\begin{array}{c}\mathbf{4} \\
0.3 \%\end{array}$ & $\begin{array}{c}34 \\
2.6 \%\end{array}$ & $\begin{array}{c}\mathbf{5 1} \\
3.9 \%\end{array}$ & $\begin{array}{c}\mathbf{5} \\
0.4 \%\end{array}$ & $\begin{array}{c}6 \\
0.5 \%\end{array}$ & $\begin{array}{c}\mathbf{3} \\
0.2 \%\end{array}$ & \\
\hline & $\begin{array}{c}12 \\
0.9 \%\end{array}$ & $\begin{array}{c}\mathbf{2 4} \\
1.8 \%\end{array}$ & $\begin{array}{c}\mathbf{2 2} \\
1.7 \%\end{array}$ & $\begin{array}{c}17 \\
1.3 \%\end{array}$ & $\begin{array}{c}\mathbf{5} \\
0.4 \%\end{array}$ & $\begin{array}{c}\mathbf{9 8} \\
7.5 \%\end{array}$ & $\begin{array}{c}13 \\
1.0 \%\end{array}$ & $\begin{array}{c}27 \\
2.1 \%\end{array}$ & \\
\hline & $\begin{array}{c}18 \\
1.4 \%\end{array}$ & $\begin{array}{c}13 \\
1.0 \% \\
\end{array}$ & $\begin{array}{c}\mathbf{2 8} \\
2.2 \% \\
\end{array}$ & $\begin{array}{c}\mathbf{4} \\
0.3 \%\end{array}$ & $\begin{array}{c}7 \\
0.5 \%\end{array}$ & $\begin{array}{c}13 \\
1.0 \%\end{array}$ & $\begin{array}{c}105 \\
8.1 \%\end{array}$ & $\begin{array}{c}12 \\
0.9 \%\end{array}$ & \\
\hline & $\begin{array}{c}\mathbf{1 8} \\
1.4 \%\end{array}$ & $\begin{array}{c}8 \\
0.6 \% \\
\end{array}$ & $\begin{array}{c}\mathbf{6} \\
0.5 \%\end{array}$ & $\begin{array}{c}\mathbf{1} \\
0.1 \%\end{array}$ & $\begin{array}{c}\mathbf{1} \\
0.1 \%\end{array}$ & $\begin{array}{c}29 \\
2.2 \%\end{array}$ & $\begin{array}{c}13 \\
1.0 \%\end{array}$ & \begin{tabular}{|c|}
81 \\
$6.2 \%$ \\
\end{tabular} & $\begin{array}{l}51.6 \\
48 .\end{array}$ \\
\hline & & & & & & & & & \\
\hline
\end{tabular}

Figure 5.8: Confusion matrix for top- 1 event prediction with a tolerance of 0.0 on the Volleyball Dataset. The rightmost column shows the precision and the bottom row shows the recall per class in percentages. The green values represent the percentages of the correct predictions by the model whereas the red values show the percentages of the incorrect predictions. For example, recall for $r$-set is $41.7 \%$ and the its precision is $51.3 \%$ which means out of all the $r$-set events, $41.7 \%$ were correctly classified and out of all the $r$-set predictions made by the model, $51.3 \%$ were correct. The blue rectangle in the bottom right corresponds to the overall accuracy. 


\begin{tabular}{|c|c|c|c|c|c|c|c|c|c|}
\hline & \multicolumn{9}{|c|}{ Confusion Matrix } \\
\hline & $\begin{array}{c}112 \\
8.6 \%\end{array}$ & $\begin{array}{c}15 \\
1.2 \%\end{array}$ & $\begin{array}{c}7 \\
0.5 \%\end{array}$ & $\begin{array}{c}\mathbf{0} \\
0.0 \%\end{array}$ & $\begin{array}{c}\mathbf{2} \\
0.2 \%\end{array}$ & $\begin{array}{c}\mathbf{5} \\
0.4 \%\end{array}$ & $\begin{array}{c}\mathbf{5} \\
0.4 \%\end{array}$ & $\begin{array}{c}12 \\
0.9 \%\end{array}$ & $\begin{array}{l}70.9 \\
29.1\end{array}$ \\
\hline r-spike & $\begin{array}{c}13 \\
1.0 \%\end{array}$ & \begin{tabular}{|c|}
113 \\
$8.7 \%$
\end{tabular} & $\begin{array}{c}\mathbf{1 5} \\
1.2 \%\end{array}$ & $\begin{array}{c}\mathbf{4} \\
0.3 \%\end{array}$ & $\begin{array}{c}\mathbf{5} \\
0.4 \%\end{array}$ & $\begin{array}{c}15 \\
1.2 \%\end{array}$ & $\begin{array}{c}\mathbf{5} \\
0.4 \%\end{array}$ & $\begin{array}{c}\mathbf{9} \\
0.7 \%\end{array}$ & $\begin{array}{l}63.1 \% \\
36.9 \%\end{array}$ \\
\hline r-pass & $\begin{array}{c}25 \\
1.9 \%\end{array}$ & $\begin{array}{c}9 \\
0.7 \%\end{array}$ & $\begin{array}{c}\mathbf{1 4 4} \\
11.1 \%\end{array}$ & $\begin{array}{c}\mathbf{5} \\
0.4 \%\end{array}$ & $\begin{array}{c}14 \\
1.1 \%\end{array}$ & $\begin{array}{c}16 \\
1.2 \%\end{array}$ & $\begin{array}{c}\mathbf{6} \\
0.5 \%\end{array}$ & $\begin{array}{c}7 \\
0.5 \%\end{array}$ & \\
\hline inpoint & $\begin{array}{c}1 \\
0.1 \%\end{array}$ & $\begin{array}{c}\mathbf{2} \\
0.2 \%\end{array}$ & $\begin{array}{c}3 \\
0.2 \%\end{array}$ & $\begin{array}{c}\mathbf{5 6} \\
4.3 \%\end{array}$ & $\begin{array}{c}\mathbf{2} \\
0.2 \%\end{array}$ & $\begin{array}{c}\mathbf{4} \\
0.3 \%\end{array}$ & $\begin{array}{c}1 \\
0.1 \%\end{array}$ & $\begin{array}{c}1 \\
0.1 \%\end{array}$ & \\
\hline winpo & $\begin{array}{c}2 \\
0.2 \%\end{array}$ & $\begin{array}{c}\mathbf{4} \\
0.3 \%\end{array}$ & $\begin{array}{c}\mathbf{4} \\
0.3 \%\end{array}$ & $\begin{array}{c}3 \\
0.2 \%\end{array}$ & $\begin{array}{c}\mathbf{6 7} \\
5.2 \%\end{array}$ & $\begin{array}{c}\mathbf{5} \\
0.4 \%\end{array}$ & $\begin{array}{c}\mathbf{4} \\
0.3 \%\end{array}$ & $\begin{array}{c}\mathbf{3} \\
0.2 \%\end{array}$ & \\
\hline & $\begin{array}{c}10 \\
0.8 \%\end{array}$ & $\begin{array}{c}9 \\
0.7 \%\end{array}$ & $\begin{array}{c}\mathbf{1 7} \\
1.3 \%\end{array}$ & $\begin{array}{c}10 \\
0.8 \%\end{array}$ & $\begin{array}{c}\mathbf{4} \\
0.3 \%\end{array}$ & $\begin{array}{c}157 \\
12.1 \%\end{array}$ & $\begin{array}{c}10 \\
0.8 \%\end{array}$ & $\begin{array}{c}\mathbf{9} \\
0.7 \%\end{array}$ & \\
\hline & $\begin{array}{c}11 \\
0.8 \%\end{array}$ & $\begin{array}{c}\mathbf{8} \\
0.6 \%\end{array}$ & $\begin{array}{c}11 \\
0.8 \%\end{array}$ & $\begin{array}{c}\mathbf{4} \\
0.3 \%\end{array}$ & $\begin{array}{c}1 \\
0.1 \%\end{array}$ & $\begin{array}{c}11 \\
0.8 \%\end{array}$ & $\begin{array}{c}137 \\
10.5 \%\end{array}$ & $\begin{array}{c}12 \\
0.9 \%\end{array}$ & \\
\hline & $\begin{array}{c}13 \\
1.0 \%\end{array}$ & $\begin{array}{c}\mathbf{8} \\
0.6 \%\end{array}$ & $\begin{array}{c}3 \\
0.2 \%\end{array}$ & $\begin{array}{c}\mathbf{0} \\
0.0 \%\end{array}$ & $\begin{array}{c}\mathbf{1} \\
0.1 \%\end{array}$ & $\begin{array}{c}7 \\
0.5 \%\end{array}$ & $\begin{array}{c}\mathbf{9} \\
0.7 \%\end{array}$ & $\begin{array}{c}113 \\
8.7 \%\end{array}$ & \\
\hline & & & & 830 & & & & & \\
\hline
\end{tabular}

Figure 5.9: Confusion matrix for top- 2 event prediction with a tolerance of 0.15 on the Volleyball Dataset. The rightmost column shows the precision and the bottom row shows the recall per class in percentages. The green values represent the percentages of the correct predictions by the model whereas the red values show the percentages of the incorrect predictions. For example, recall for $r$-set is $59.9 \%$ and the its precision is $70.9 \%$ which means out of all the $r$-set events, $59.9 \%$ were correctly classified and out of all the $r$-set predictions made by the model, $70.9 \%$ were correct. The blue rectangle in the bottom right corresponds to the overall accuracy. 

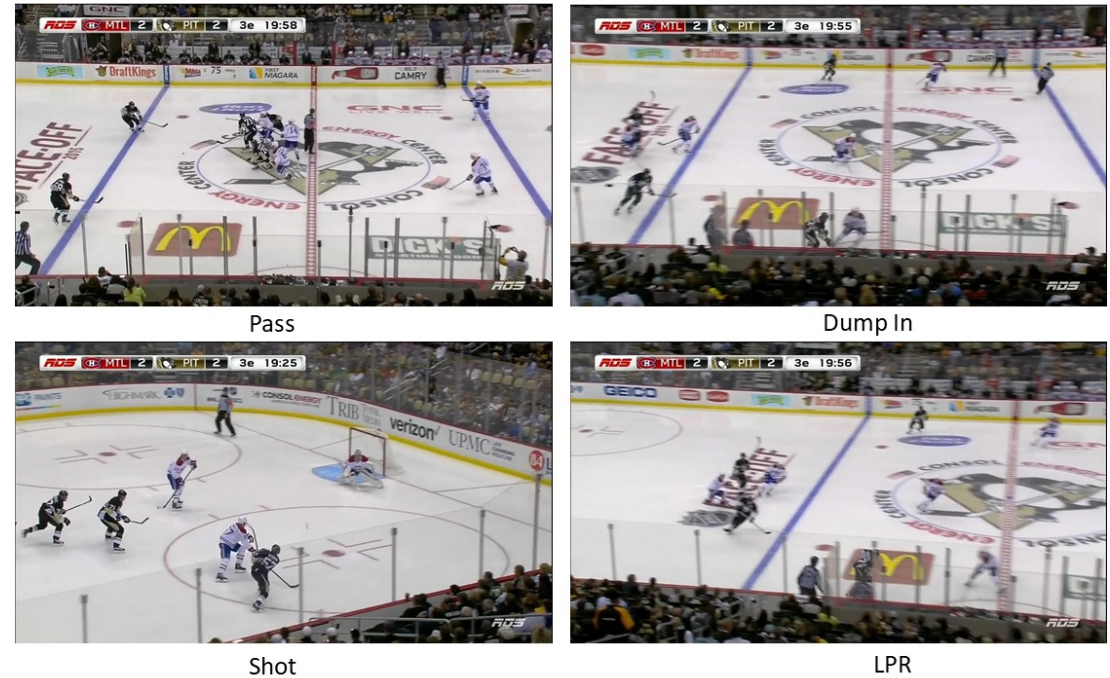

Figure 5.10: Qualitative results. The correct predictions made by our model with a tolerance of 0.0 on the Ice Hockey Dataset.

between them if we only consider the player features. But our model successfully identified the correct event which shows the importance of encoding the frame features. The dataset has many such examples where the team jerseys are very similar and our model successfully distinguished and classified them. Figure 5.14 shows the scenarios where our model made a wrong prediction with a tolerance of 0.0 but a correct prediction with a tolerance of 0.15 . There are several interesting observations for this figure. In most cases, the model was confused between left and right. For example, in the first image, left spike was confused with right spike. Moreover, the probability difference is very small in most cases which shows that the model is highly confident about the true events but it had confusion between the side of the court because we do not have any mechanism to distinguish between the left and the right teams. The events that are considered for this dataset can be merged into 4 events and sub divided into left and right. As a result, it is important that we incorporate some sort of mechanism to distinguish the team sides. Homography features could be an important cue to solve this ambiguity as they incorporate the spatial player arrangements into the model. Lastly, Figure 5.15 shows some examples where our model failed. 


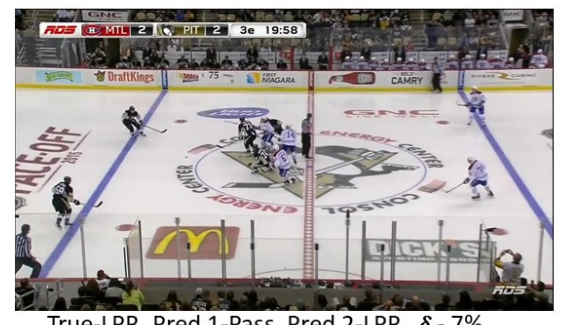

True-LPR, Pred 1-Pass, Pred 2-LPR, $\delta-7 \%$

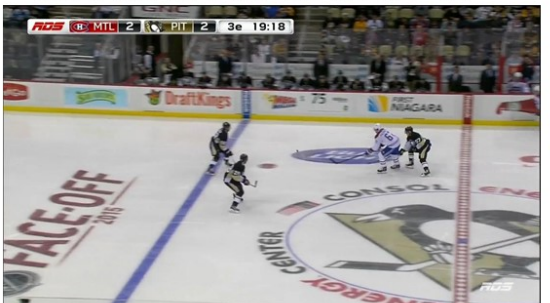

True-Dump In, Pred 1-Pass, Pred 2-Dump In, $\delta$ - 3\%
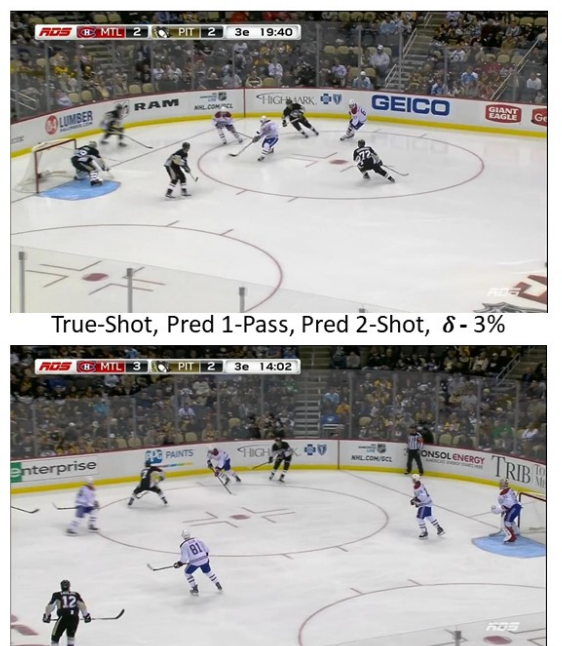

True-Dump Out, Pred 1-Pass, Pred 2-Dump Out, $\delta$ - 10\%

Figure 5.11: Qualitative results. The correct predictions made by our model with a tolerance of 0.15 on the Ice Hockey Dataset. True refers to the actual class, Pred 1 and Pred 2 refer to the prediction with a tolerance of 0.0 and 0.15 respectively and $\delta$ refers to the difference in probabilities between the two predictions.
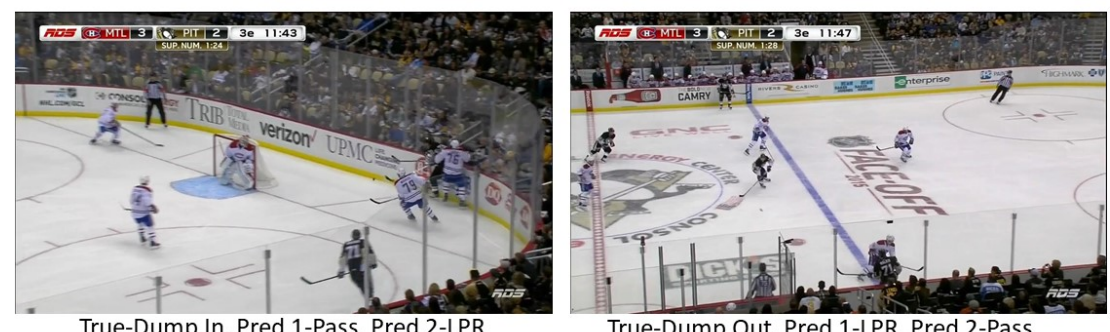

True-Dump In, Pred 1-Pass, Pred 2-LPR

True-Dump Out, Pred 1-LPR, Pred 2-Pass
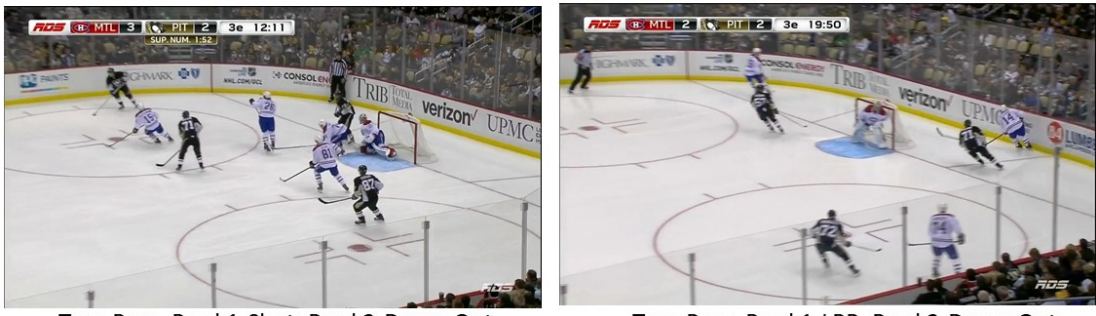

True-Pass, Pred 1-Shot, Pred 2-Dump Out

True-Pass, Pred 1-LPR, Pred 2-Dump Out

Figure 5.12: Qualitative results. The images show the cases where our model failed on the Ice Hockey Dataset. 


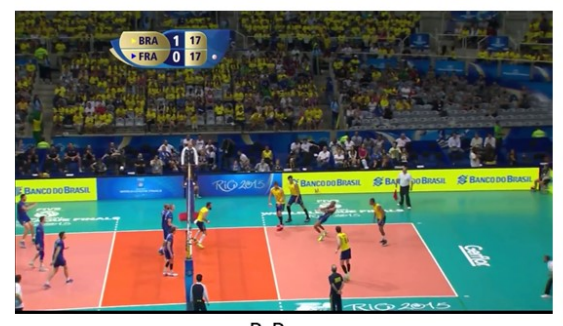

R-Pass

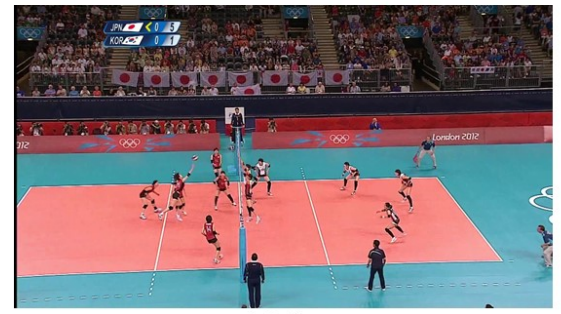

L-Spike
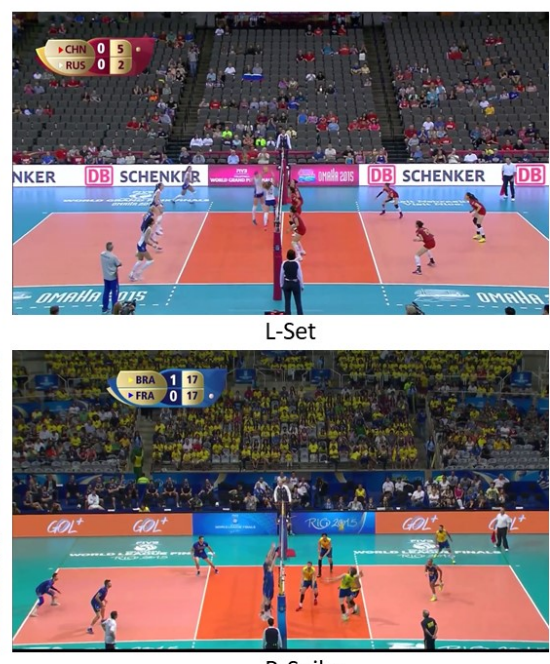

R-Spike

Figure 5.13: Qualitative results. The correct predictions made by our model with a tolerance of 0.0 on the Volleyball Dataset.
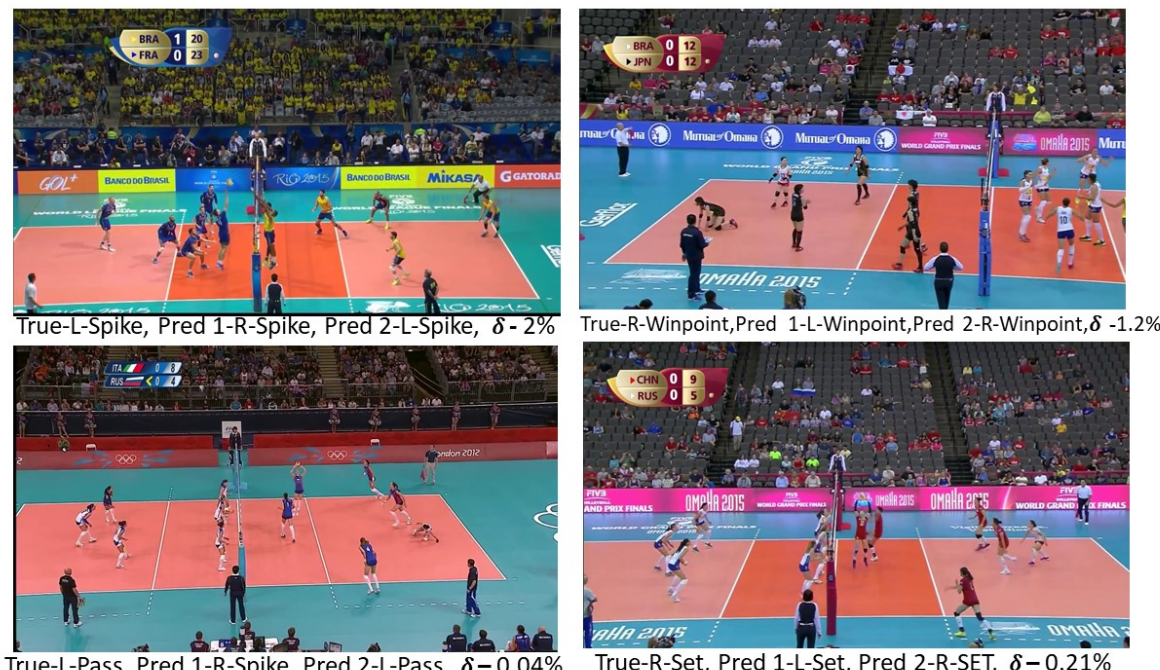

Figure 5.14: Qualitative results. The correct predictions made by our model with a tolerance of 0.15 on the Volleyball Dataset. True refers to the actual class, Pred 1 and Pred 2 refer to the prediction with a tolerance of 0.0 and 0.15 respectively and $\delta$ refers to the difference in probabilities between the two predictions. 

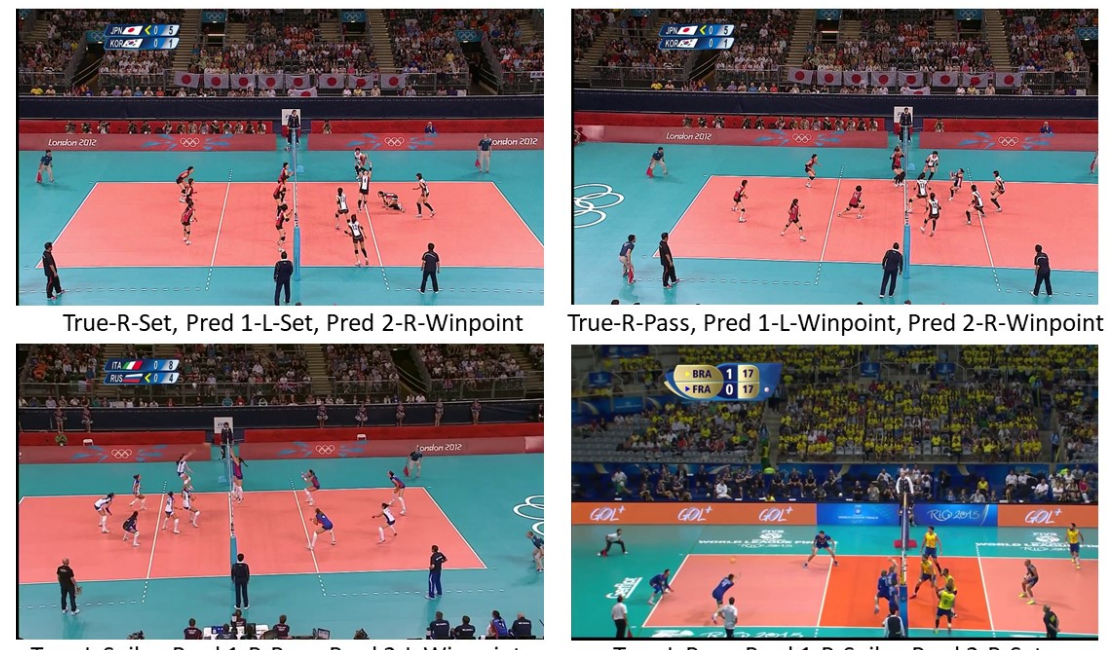

True-L-Spike, Pred 1-R-Pass, Pred 2-L-Winpoint

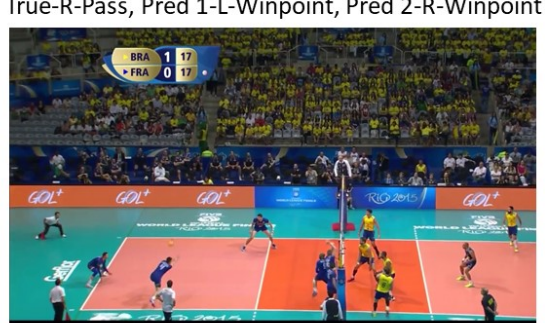

True-L-Pass, Pred 1-R-Spike, Pred 2-R-Set

Figure 5.15: Qualitative results. The figures show the cases where our model failed on the Volleyball Dataset. 


\section{Chapter 6}

\section{Conclusion}

In this thesis, we proposed a deep learning model to classify group activities in ice hockey. We have shown that feature aggregation is essential in determining the game events. We have looked at five puck possession events and shown that they can be classified without the need of explicit labeling for individual actions or puck information. We have proposed a fusion model to combine deep features from different sources and shallow features. We have also applied our partial model to a Volleyball Dataset and received competitive results.

Our problem and solution are very generalized and there are many possible future extensions. Any types of features can be given as inputs to our fusion model and combined to see how that influences the accuracy. We would like to do a more detailed analysis of the homography features because the weights that we have computed might be informative about the possible puck location in the rink. Rather than using the puck location to classify the events, one possible extension could be to use the homography features and events knowledge to locate the puck. Currently we do not have the evaluation results of our model on the Volleyball Dataset due to lack of homography information. We would like to build the homography matrices for the volleyball games and extract the homography features to run our final model on this dataset.

We have used the AlexNet architecture [34] for the deep feature extraction which is quite old. So one important extension of our work would be to try more recent CNNs such as ResNet [24] or VGGNet [54] and compare with AlexNet. Fu- 
ture work will also focus on incorporating player motion and 3D pose information into this model. These features might be essential cues for motion-based events such as pass and shot or determining the appearance of the players. In this paper, we have looked at a subset of many possible puck possession events. We would also like to classify other events such as goal and carry in. Deep learning models require a huge number of training examples and our dataset was quite small. We would like to add more games and increase the dataset size especially for the less frequent classes so that the dataset is more balanced. Other possible extensions might include finding the motion and position of the hockey sticks with respect to the players and taking advantage of gaze information of the players. 


\section{Bibliography}

[1] Convolutional neural networks for visual recognition, . URL http://cs231n.github.io/convolutional-networks/, $\rightarrow$ pages 13

[2] Example image of alexnet, . URL https://ischlag.github.io/images/alexnet.png $\rightarrow$ pages ix, 14

[3] Popular cnn architectures, . URL https://adeshpande3.github.io/adeshpande3.github.io/ The-9-Deep-Learning-Papers-You-Need-To-Know-About.html. $\rightarrow$ pages 15

[4] Example image of a long short term memory network (lstm). URL https: //devblogs.nvidia.com/parallelforall/wp-content/uploads/2016/03/LSTM.png $\rightarrow$ pages ix, 17

[5] Example image of a recurrent neural network (rnn). URL http://colah.github. io/posts/2015-08-Understanding-LSTMs/img/RNN-unrolled.png. $\rightarrow$ pages ix, 17

[6] M. Abadi, A. Agarwal, P. Barham, E. Brevdo, Z. Chen, C. Citro, G. S. Corrado, A. Davis, J. Dean, M. Devin, et al. Tensorflow: Large-scale machine learning on heterogeneous distributed systems. arXiv preprint arXiv:1603.04467, 2016. $\rightarrow$ pages 24, 27

[7] M. R. Amer, P. Lei, and S. Todorovic. HIRF: Hierarchical random field for collective activity recognition in videos. In European Conference on Computer Vision (ECCV), 2014. $\rightarrow$ pages 2,8

[8] T. Bagautdinov, A. Alahi, F. Fleuret, P. Fua, and S. Savarese. Social scene understanding: End-to-end multi-person action localization and collective activity recognition. In Proceedings of the Conference on Computer Vision and Pattern Recognition (CVPR), 2017. $\rightarrow$ pages 8, 10, 37, 39 
[9] F. Caba Heilbron, W. Barrios, V. Escorcia, and B. Ghanem. SCC: Semantic context cascade for efficient action detection. In Proceedings of the IEEE Conference on Computer Vision and Pattern Recognition (CVPR), 2017. $\rightarrow$ pages 12

[10] D. Cervone, A. DAmour, L. Bornn, and K. Goldsberry. POINTWISE: Predicting points and valuing decisions in real time with nba optical tracking data. In 8th Annual MIT Sloan Sports Analytics Conference, 2014. $\rightarrow$ pages 9

[11] J. Chen and J. J. Little. Where should cameras look at soccer games: Improving smoothness using the overlapped hidden markov model. In Computer Vision and Image Understanding, 2017. $\rightarrow$ pages 11, 20

[12] J. Chen, H. M. Le, P. Carr, Y. Yue, and J. J. Little. Learning online smooth predictors for realtime camera planning using recurrent decision trees. In Proceedings of the IEEE Conference on Computer Vision and Pattern Recognition (CVPR), 2016. $\rightarrow$ pages 1

[13] W. Choi and S. Savarese. A unified framework for multi-target tracking and collective activity recognition. European Conference on Computer Vision $(E C C V), 2012$. $\rightarrow$ pages 2

[14] W. Choi and S. Savarese. Understanding collective activities of people from videos. IEEE Transactions on Pattern Analysis and Machine Intelligence, 36 (6):1242-1257, 2014. $\rightarrow$ pages 2,8

[15] W. Choi, K. Shahid, and S. Savarese. What are they doing? : Collective activity classification using spatio-temporal relationship among people. In Visual Surveillance Workshop, ICCV, 2009. $\rightarrow$ pages 1,8

[16] Z. Deng, A. Vahdat, H. Hu, and G. Mori. Structure inference machines: Recurrent neural networks for analyzing relations in group activity recognition. In Proceedings of the IEEE Conference on Computer Vision and Pattern Recognition (CVPR), 2016. $\rightarrow$ pages 8,12

[17] J. Donahue, L. Anne Hendricks, S. Guadarrama, M. Rohrbach, S. Venugopalan, K. Saenko, and T. Darrell. Long-term recurrent convolutional networks for visual recognition and description. In Proceedings of the IEEE Conference on Computer Vision and Pattern Recognition (CVPR), 2015. $\rightarrow$ pages 12, 18

[18] E. Dubrofsky. Homography estimation. In Masters Thesis, University of British Columbia (Vancouver), Kelowna, BC, Canada, 2009. $\rightarrow$ pages 20 
[19] R. Girshick. Fast r-cnn. In Proceedings of the IEEE International Conference on Computer Vision (ICCV), 2015. $\rightarrow$ pages 15

[20] R. Girshick, J. Donahua, T. Darrell, and J. Malik. Rich feature hierarchies for accurate object detection and semantic segmentation. In Proceedings of the IEEE conference on Computer Vision and Pattern Recognition (CVPR), 2014. $\rightarrow$ pages 15

[21] I. Goodfellow, J. Pouget-Abadie, M. Mirza, Xu, D. Warde-Farley, S. Ozair, A. Courville, and Y. Bengio. Generative adversarial nets. In Advances in Neural Information Processing Systems (NIPS), 2014. $\rightarrow$ pages 15

[22] A. Graves and N. Jaitly. Towards end-to-end speech recognition with recurrent neural networks. In International Conference on Machine Learning (ICML), 2014. $\rightarrow$ pages 12

[23] A. Gupta, J. Little, and R. Woodham. Using line and ellipse features for rectification of broadcast hockey video. In Computer and Robot Vision (CRV), 2011. $\rightarrow$ pages 20

[24] K. He, X. Zhang, S. Ren, and J. Sun. Deep residual learning for image recognition. In Proceedings of the IEEE conference on computer vision and pattern recognition $(C V P R), 2016$. $\rightarrow$ pages 15,47

[25] A. Hilton, J.-Y. Guillemaut, J. Kilner, O. Grau, and G. Thomas. Free-viewpoint video for TV sport production. In Image and Geometry Processing for 3-D Cinematography, pages 77-106. 2010. $\rightarrow$ pages 1

[26] S. Hochreiter and J. Schmidhuber. Long short-term memory. Neural Computation, 9(8):1735-1780, 1997. $\rightarrow$ pages 12,16

[27] N. Homayounfar, S. Fidler, and R. Urtasun. Soccer field localization from a single image. In arXiv preprint arXiv:1604.02715, 2016. $\rightarrow$ pages 11

[28] M. S. Ibrahim, S. Muralidharan, Z. Deng, A. Vahdat, and G. Mori. A hierarchical deep temporal model for group activity recognition. In Proceedings of the IEEE Conference on Computer Vision and Pattern Recognition (CVPR), 2016. $\rightarrow$ pages 8, 10, 18, 19, 24, 28, 37, 39

[29] M. Jaderberg, K. Simonyan, and Z. A. Spatial transformer networks. In Advances in Neural Information Processing Systems (NIPS), 2015. $\rightarrow$ pages 15 
[30] A. Karpathy and L. Fei-Fei. Deep visual-semantic alignments for generating image descriptions. In Proceedings of the IEEE Conference on Computer Vision and Pattern Recognition (CVPR), 2015. $\rightarrow$ pages 12

[31] A. Karpathy, G. Toderici, S. Shetty, T. Leung, R. Sukthankar, and L. Fei-Fei. Large-scale video classification with convolutional neural networks. In Proceedings of the IEEE Conference on Computer Vision and Pattern Recognition (CVPR), 2014. $\rightarrow$ pages 12, 22

[32] D. Kingma and J. Ba. Adam: A method for stochastic optimization. arXiv preprint arXiv:1412.6980, 2014. $\rightarrow$ pages 27

[33] Y. Kong, W. Hu, X. Zhang, H. Wang, and Y. Jia. Learning group activity in soccer videos from local motion. In Proceedings of Asian Conference on Computer Vision, 2009. $\rightarrow$ pages 11

[34] A. Krizhevsky, I. Sutskever, and G. E. Hinton. Imagenet classification with deep convolutional neural networks. In Advances in Neural Information Processing Systems (NIPS), 2012. $\rightarrow$ pages 12, 14, 15, 18, 47

[35] T. Lan, L. Sigal, and G. Mori. Social roles in hierarchical models for human activity recognition. In Proceedings of the IEEE Conference on Computer Vision and Pattern Recognition (CVPR), pages 1354-1361, 2012. $\rightarrow$ pages 8

[36] T. Lan, Y. Wang, W. Yang, S. N. Robinovitch, and G. Mori. Discriminative latent models for recognizing contextual group activities. IEEE Transactions on Pattern Analysis and Machine Intelligence, 34(8):1549-1562, 2012. $\rightarrow$ pages 2,8

[37] J. Liu, X. Tong, W. Li, T. Wang, Y. Zhang, and H. Wang. Automatic player detection, labeling and tracking in broadcast soccer video. In Pattern Recognition Letters, 2009. $\rightarrow$ pages 11

[38] J. Liu, P. Carr, R. T. Collins, and Y. Liu. Tracking sports players with context-conditioned motion models. In Proceedings of the IEEE Conference on Computer Vision and Pattern Recognition (CVPR), 2013. $\rightarrow$ pages 1

[39] W.-L. Lu and J. J. Little. Simultaneous tracking and action recognition using the pca-hog descriptor. In The 3rd Canadian Conference on Computer and Robot Vision, 2006. $\rightarrow$ pages 9

[40] W.-L. Lu and J. J. Little. Tracking and recognizing actions at a distance. In Proceedings of the ECCV Workshop on Computer Vision Based Analysis in Sport Environments, 2006. $\rightarrow$ pages 
[41] W.-L. Lu, K. Okuma, and J. J. Little. Tracking and recognizing actions of multiple hockey players using the boosted particle filter. In Image and Vision Computing, 2009. $\rightarrow$ pages 9

[42] B. Macdonald. An improved adjusted plus-minus statistic for NHL players. In Proceedings of the MIT Sloan Sports Analytics Conference, 2011. $\rightarrow$ pages 9

[43] T. B. Moeslund, G. Thomas, and A. Hilton. Computer vision in sports. In Springer, 2014. $\rightarrow$ pages 1

[44] V. Ramanathan, B. Yao, and L. Fei-Fei. Social role discovery in human events. In Proceedings of the IEEE Conference on Computer Vision and Pattern Recognition (CVPR), 2013. $\rightarrow$ pages 8

[45] V. Ramanathan, J. Huang, S. Abu-El-Haija, A. Gorban, K. Murphy, and L. Fei-Fei. Detecting events and key actors in multi-person videos. In Proceedings of the IEEE Conference on Computer Vision and Pattern Recognition $(C V P R), 2016$. $\rightarrow$ pages 8, 11, 12

[46] S. Ren, K. He, R. Girshick, and J. Sun. Faster r-cnn: Towards real-time object detection with region proposal networks. In Advances in Neural Information Processing Systems (NIPS), 2015. $\rightarrow$ pages 15

[47] M. Ryoo and J. Aggarwal. Recognition of composite human activities through context-free grammer based representation. In Proceedings of the IEEE Conference on Computer Vision and Pattern Recognition (CVPR), 2006. $\rightarrow$ pages 8

[48] H. Sak, A. W. Senior, and F. Beaufays. Long short-term memory recurrent neural network architectures for large scale acoustic modeling. In Proceedings of the Annual Conference of International Speech Communication Association (INTERSPEECH), 2014. $\rightarrow$ pages 23

[49] O. Schulte, M. Khademi, S. Gholami, Z. Zhao, M. Javan, and P. Desaulniers. A markov game model for valuing actions, locations, and team performance in ice hockey. Data Mining and Knowledge Discovery, pages 1-23, 2017. $\rightarrow$ pages 9

[50] M. Schuster and K. K. Paliwal. Bidirectional recurrent neural networks. IEEE Transactions on Signal Processing, 45(11), pages 2673-2681, 1997. $\rightarrow$ pages 16, 22 
[51] T. Shu, D. Xie, B. Rothrock, S. Todorovic, and S. Chun Zhu. Joint inference of groups, events and human roles in aerial videos. In Proceedings of the IEEE Conference on Computer Vision and Pattern Recognition (CVPR), 2015. $\rightarrow$ pages 8

[52] T. Shu, S. Todorovic, and S.-C. Zhu. Cern: Confidence-energy recurrent network for group activity recognition. In Proceedings of the Conference on Computer Vision and Pattern Recognition (CVPR), 2017. $\rightarrow$ pages 8

[53] K. Simonyan and A. Zisserman. Two-stream convolutional networks for action recognition in videos. In Advances in Neural Information Processing Systems (NIPS), 2014. $\rightarrow$ pages 12

[54] K. Simonyan and A. Zisserman. Very deep convolutional networks for large-scale image recognition. In arXiv preprint arXiv:1409.1556., 2014. $\rightarrow$ pages 15,47

[55] K. Soomro, A. R. Zamir, and M. Shah. UCF101: A dataset of 101 human actions classes from videos in the wild. arXiv preprint arXiv:1212.0402, 2012. $\rightarrow$ pages 27

[56] C. Szegedy, W. Liu, Y. Jia, P. Sermanet, S. Reed, D. Anguelov, D. Erhan, V. Vanhoucke, and A. Rabinovich. Going deeper with convolutions. In Proceedings of the IEEE Conference on Computer Vision and Pattern Recognition (CVPR), 2015. $\rightarrow$ pages 15

[57] G. Thomas. Real-time camera tracking using sports pitch markings. In Journal of Real-Time Image Processing, 2007. $\rightarrow$ pages 20

[58] D. Tran, L. Bourdev, R. Fergus, L. Torresani, and M. Paluri. Learning spatiotemporal features with 3D convolutional networks. In IEEE International Conference on Computer Vision (ICCV), 2015. $\rightarrow$ pages 27, $28,34,39$

[59] T. Tsunoda, Y. Komori, M. Matsugu, and T. Harada. Football action recognition using hierarchical lstm. In 3rd IEEE International Workshop on Computer Vision in Sports (CVsports), 2017. $\rightarrow$ pages 11

[60] O. Vinyals, A. Toshev, S. Bengio, and D. Erhan. Show and tell: A neural image caption generator. In Proceedings of the IEEE Conference on Computer Vision and Pattern Recognition (CVPR), 2015. $\rightarrow$ pages 12 
[61] M. Wang, B. Ni, and X. Yang. Recurrent modeling of interaction context for collective activity recognition. In Proceedings of the Conference on Computer Vision and Pattern Recognition (CVPR), 2017. $\rightarrow$ pages 8

[62] Y. Wang and G. Mori. Max-margin hidden conditional random fields for human action recognition. In Proceedings of the Conference on Computer Vision and Pattern Recognition (CVPR), 2009. $\rightarrow$ pages 8

[63] X. Wei, P. Lucey, S. Morgan, and S. Sridharan. Forecasting the next shot location in tennis using fine-grained spatiotemporal tracking data. IEEE Transactions on Knowledge and Data Engineering, 28(11):2988-2997, 2016. $\rightarrow$ pages 1

[64] S. Yeung, O. Russakovsky, G. Mori, and L. Fei-Fei. End-to-end learning of action detection from frame glimpses in videos. In Proceedings of the IEEE Conference on Computer Vision and Pattern Recognition (CVPR), 2016. $\rightarrow$ pages 8

[65] J. Yue-Hei Ng, M. Hausknecht, S. Vijayanarasimhan, O. Vinyals, R. Monga, and G. Toderici. Beyond short snippets: Deep networks for video classification. In Proceedings of the IEEE Conference on Computer Vision and Pattern Recognition (CVPR), 2015. $\rightarrow$ pages 9

[66] M. Zeng, L. T. Nguyen, B. Yu, O. J. Mengshoel, J. Zhu, P. Wu, and J. Zhang. Convolutional neural networks for human activity recognition using mobile sensors. In 6th International Conference on Mobile Computing, Applications and Services (MobiCASE), 2014. $\rightarrow$ pages 19 